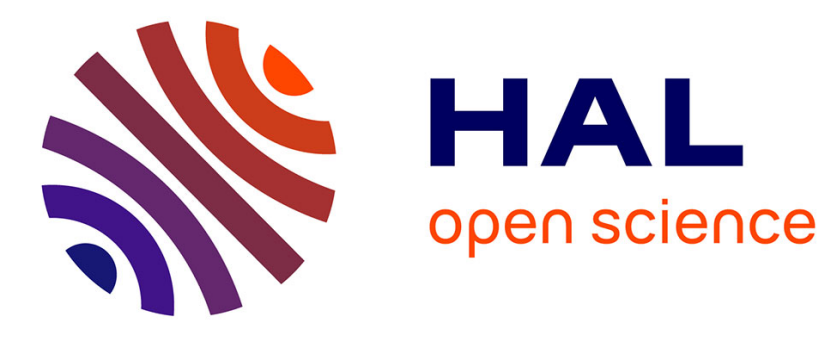

\title{
Image Zoom Completion
}

\author{
Moncef Hidane, Mireille El Gheche, Jean-François Aujol, Yannick \\ Berthoumieu, Charles Deledalle
}

\section{To cite this version:}

Moncef Hidane, Mireille El Gheche, Jean-François Aujol, Yannick Berthoumieu, Charles Deledalle. Image Zoom Completion. IEEE Transactions on Image Processing, 2016, 25 (8), pp.3505-3517. 10.1109/TIP.2016.2571061 . hal-01253124v2

\section{HAL Id: hal-01253124 \\ https://hal.science/hal-01253124v2}

Submitted on 30 May 2016

HAL is a multi-disciplinary open access archive for the deposit and dissemination of scientific research documents, whether they are published or not. The documents may come from teaching and research institutions in France or abroad, or from public or private research centers.
L'archive ouverte pluridisciplinaire HAL, est destinée au dépôt et à la diffusion de documents scientifiques de niveau recherche, publiés ou non, émanant des établissements d'enseignement et de recherche français ou étrangers, des laboratoires publics ou privés. 


\title{
Image Zoom Completion
}

\author{
Moncef Hidane, Mireille El Gheche, Jean-François Aujol, Yannick Berthoumieu, Charles-Alban Deledalle
}

\begin{abstract}
We consider the problem of recovering a highresolution image from a pair consisting of a complete lowresolution image and a high-resolution but incomplete one. We refer to this task as the image zoom completion problem. After discussing possible contexts in which this setting may arise, we introduce a nonlocal regularization strategy, giving full details concerning the numerical optimization of the corresponding energy, and discussing its benefits and shortcomings. We also derive two total variation-based algorithms and evaluate the performance of the proposed methods on a set of natural and textured images. We compare the results we get with those obtained with two recent state-of-the-art single-image superresolution algorithms.
\end{abstract}

\section{INTRODUCTION}

Image restoration problems are ubiquitous in the field of image processing [1]. Therein, the goal is to estimate an underlying image from a set of related, degraded, and possibly incomplete measurements. The first step towards this goal is the identification of the imaging device parameters, as well as those pertaining to the scene being imaged. Considering a linear forward model, either known a priori or properly estimated, with additive noise, the restoration problem can be cast as an inverse problem where the goal is to recover an image $\mathbf{f} \in \mathbb{R}^{n}, n \geqslant 1$, related to measurements $\mathbf{y} \in \mathbb{R}^{p}$, $p \leqslant n$, through the forward model

$$
\mathbf{y}=\mathbf{A f}+\boldsymbol{\eta}
$$

where $\mathbf{A} \in \mathbb{R}^{p \times n}$ is a known linear operator and $\boldsymbol{\eta}$ is unknown and accounts for both sensing and modeling errors.

Depending on the nature of the matrix $\mathbf{A}$ in (1), different restoration problems are encountered. In all cases, the very nature of imaging devices leads to problems of the form (1) which are either undetermined $(p<n)$ or ill-conditioned (the condition number of $\mathbf{A}$ is very high). A common strategy to deal with such ill-posed inverse problems is through the variational approach. In this setting, specific prior information about the sought-after image is selected by choosing a penalty function $J$. An estimated image $\hat{\mathbf{f}}$ is then obtained by minimizing an overall cost function imposing a trade-off between prior information (through $J$ ) and a data fidelity term

Moncef Hidane is with INSA Centre Val de Loire and with Laboratoire d'Informatique de l'Université de Tours.

Mireille El Gheche is with the IMS and IMB laboratories of Université de Bordeaux.

Jean-François Aujol and Charles Deledalle are with IMB CNRS-Université de Bordeaux and Institut Polytechnique de Bordeaux.

Yannick Berthoumieu is with Institut Polytechnique de Bordeaux and with the IMS Laboratory.

This study has been carried out with financial support from the French State, managed by the French National Research Agency (ANR) in the frame of the "Investments for the future" Programme IdEx Bordeaux - CPU (ANR10-IDEX-03-02).

J.-F. Aujol acknowledges the support of the Institut Universitaire de France. controlling the discrepancy between the estimated image $\hat{\mathbf{f}}$ and the measurements $\mathbf{y}$ according to the forward model (1).

\section{A. Image Zoom Completion}

We study in this paper a specific restoration problem that we term image zoom completion (IZC). Here, the problem is to recover a high-resolution (HR) image from a pair consisting of a complete low-resolution (LR) image and an HR but incomplete one. To the best of our knowledge, this problem has been introduced for the first time by the authors of the present paper in [2].

The application that lead us to investigate a solution to the IZC problem concerned the estimation of petro-physical parameters through image processing techniques. In this context, a 3D computerized tomography (CT) scan of a cylindrical sample of a rock is performed. This acquisition is analyzed in order to automatically segment and classify different regions according to visual cues related to underlying petro-physical parameters. A typical example concerns the automatic classification of different textured regions, which in turn relate to different porosity levels of the underlying material. In practice, due to the large size of the sample being imaged, the particular CT system used was not able to provide enough resolution for automatic classification. In the context we were interested in, it was possible to perform a second scan, this time focusing on a specific part of the volume. This second acquisition provides incomplete but higher resolution slices of the rock sample. Now, the next step is to combine the image data coming from both acquisitions in order to obtain a complete HR volume.

The setting we have just described may also arise in the context of digital photography when one uses a digital camera to capture an image of a given scene, and then uses the optical zoom of the same camera to capture a subset of the same scene. Due to optical zoom, this second acquisition is incomplete, in the sense that it only captures a subset of the first one, but it provides higher resolution. The IZC problem is now to compute a full HR image with the aid of the complete LR and incomplete HR data. Figure 1 illustrates this setting.

The IZC problem we have just described is closely related to the classical single-image super-resolution (SISR) problem. In fact, when no subset of the HR image is available, the two problems are identical. We review in the next subsection some approaches related to the SISR problem. Due to the large body of existing literature, we concentrate on specific approaches related to our present proposal.

\section{B. SISR}

In the SISR setting, the goal is to recover an HR image from a single input LR frame. In this context, there are two 


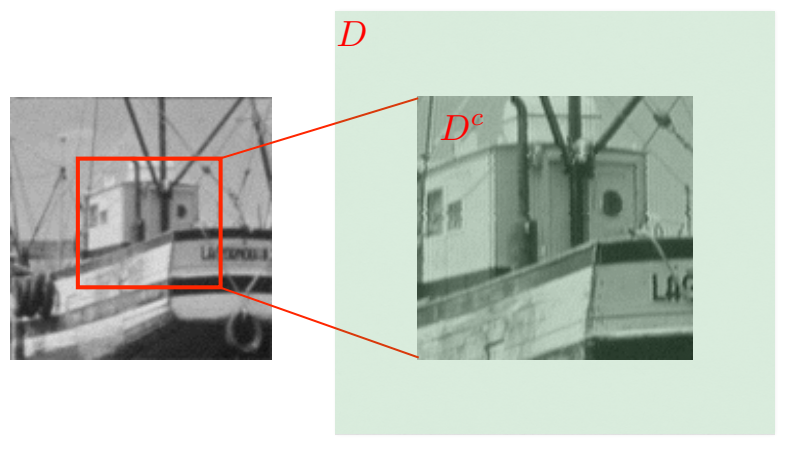

Fig. 1. Setting for the IZC problem. The left image represents a complete LR acquisition. The right image represents a zoom on a subset of the left image and is thus referred to as an incomplete HR image. The goal of IZC is to combine both acquisitions in order to complete the missing HR information.

degradation sources relating the sought-after image to the observed one: blurring and coarse sampling. A possible forward model is given by (1) with $\mathbf{A}=\mathbf{S} \circ \mathbf{H}$, where $\mathbf{H} \in \mathbb{R}^{n \times n}$ is a convolution (or more generally a linear filtering) operator and $\mathbf{S} \in \mathbb{R}^{m \times n}$ is a sub-sampling operator. This problem is underconstrained, thus SISR algorithms generally rely on various kinds of assumptions in order to estimate the true image.

The baseline method for SISR is through linear interpolation methods, especially bicubic interpolation. In order to outperform bicubic interpolation, many variational approaches exploiting smoothness and/or geometric regularity of images have been proposed (see [3] and references therein). For instance, in [4], the authors study the use of the total variation (TV) semi-norm [5] for SISR. The use of the recurrence of image patches as a cue to regularize the SISR problem has been introduced in [6]. This idea has been pushed further in [7] by restricting the search for example patches to localized regions, and in [8] by using patch redundancy across different scales. Similar ideas have been proposed for the multiframe SR setting, in particular in [9]. Approaches based on image sparsity, either in analysis or synthesis forms [10], [11] or through Gaussian mixtures [12] have also been recently considered. The application of ideas combining sparsity and self-similarity [13], [14], [15] currently leads to the best performing methods.

A different line of work involves sparse representation of LR/HR pairs of patches. This kind of approach has been initiated in [16], [17], [18] where the authors propose to infer the sparse code of each sought-after HR patch from the sparse code of its corresponding observed LR version. To enforce the invariance of LR/HR sparse codes, two coupled LR/HR dictionaries are learned from a large training data set.

\section{Organization of the Paper}

The paper is organized as follows. We formulate in Section II an observation model for the IZC setting. This leads to view the IZC problem as an inverse one whose ill-posed nature is briefly discussed. Then, we outline the general variational strategy we adopt in order to perform zoom completion.

Section III is dedicated to our main contribution. Therein, we present a nonlocal regularization strategy targeting images with possibly rich textural content. To this end, we use the recurrence of image patches at the same scale as a cue to perform zoom completion. This is done by computing patch similarities and imposing nonlocal smoothness for the superresolved image. We carry out the minimization using the recent proximal forward-backward primal-dual (FBPD) algorithm of [19].

Section IV is devoted to two TV-based regularization strategies to perform IZC. The first one consists in a TV- $\ell_{2} \mathrm{SR}$ method where the visible HR part is involved through an equality constraint. The second one is based on the recent decomposition model of [20] allowing to impose different priors on the structure and texture components of the sought-after image. For both methods, we carry out the minimization by using variable splitting and deploying the Douglas-Rachford algorithm [21] in a product-space.

We report a set of numerical experiments in Section $\mathrm{V}$, comparing the results we get with two recent state-of-the-art SISR algorithms.

Concluding remarks and directions for possible future work are outlined in Section VI. Finally, the appendix presents some background material about proximity operators, the FBPD and Douglas-Rachford algorithms, and the derivation of the algorithms presented in Section IV.

A preliminary version of this paper has been published in [2]. We bring to the attention of the reader the fact that the nonlocal regularization proposed in [2] is different from the present one. Indeed, the former is based on a nonlocal graph where each missing pixel is connected to a set of pixels that lie in the HR region, while the latter adds to the previous connections a set of connections from each pixel to its nearest neighbors in a local window. In this respect, the present construction generalizes that of [2]. We also note that the optimization algorithm used in the present paper is different from that of [2]. This longer version also includes complete details concerning the numerical optimization of the TV-based approach mentioned in [2]. Finally, the simultaneous decomposition and zoom completion method presented in Section IV-B was not present in [2].

\section{The Image Zoom Completion Problem}

The setting for IZC is a complete LR image and a corresponding incomplete HR image. Let $\mathbf{y}^{(1)} \in \mathbb{R}^{p}$ and $\mathbf{y}^{(2)} \in \mathbb{R}^{n}$, $1 \leqslant p<n$, respectively denote the lexicographical ordering of the complete LR and partial HR images. We adopt the following discrete forward model:

$$
\left\{\begin{array}{l}
\mathbf{y}^{(1)}=\mathbf{S} \mathbf{H} \mathbf{f}_{0}+\boldsymbol{\eta}_{1} \in \mathbb{R}^{p} \\
\mathbf{y}^{(2)}=\mathbf{M}\left(\mathbf{f}_{0}+\boldsymbol{\eta}_{2}\right) \in \mathbb{R}^{n}
\end{array}\right.
$$

where

- $\mathbf{f}_{0} \in \mathbb{R}^{n}$ denotes the unknown HR image;

- $\mathbf{S} \in \mathbb{R}^{p \times n}$ stands for spatial downsampling by a factor $r$ in each direction $\left(n=p r^{2}\right)$; Note that $\mathbf{S}^{\top} \in \mathbb{R}^{n \times p}$ corresponds to upsampling by the same factor and that the matrix $\mathbf{S}^{\top} \mathbf{S} \in \mathbb{R}^{n \times n}$ is diagonal with binary diagonal elements;

- $\mathbf{H} \in \mathbb{R}^{n \times n}$ accounts for spatial blurring of the image, modeled in our case by a discrete circular convolution with a known point spread function $\mathbf{h}$ : $\mathbf{H} \mathbf{x}=\mathbf{h} \circledast \mathbf{x}$; 
- $\mathbf{M}=\operatorname{diag}\left(m_{1}, \ldots, m_{n}\right) \in \mathbb{R}^{n \times n}$ is a binary mask indicating which HR pixels are observed: $m_{i}=1$, if pixel $i$ belongs to the observed area of the HR image, and $m_{i}=0$ otherwise;

- the vectors $\boldsymbol{\eta}_{1} \in \mathbb{R}^{p}$ and $\boldsymbol{\eta}_{2} \in \mathbb{R}^{n}$ are samples of a white Gaussian noise, accounting for acquisition and modeling errors.

Throughout the paper, we assume that the forward model (2) relating the HR/LR pair to the sought-after image $f_{0}$ is completely known. In particular, we discuss neither the identification of the convolution kernel nor possible registration issues when the partial zoom is performed.

Under the setting described above, the IZC problem corresponds to the recovery of an estimate $\hat{\mathbf{f}}$ of $\mathbf{f}_{0}$ from the measurements $\mathbf{y}^{(1)}$ and $\mathbf{y}^{(2)}$, according to the forward model (2). Depending on the downsampling factor $r$, the support of the blur kernel and the visible HR area, this problem can be under-determined. In all cases, the presence of the convolution operator makes it ill-conditioned.

As usual for such inverse problems in imaging [1], we formulate the estimation task in a variational setting leading to the minimization of an energy function of the form $E(\mathbf{f})=R(\mathbf{f})+D(\mathbf{f})$. The function $R$ is a regularization term forcing the solutions to have pre-specified properties, while the term $D(\mathbf{f})$ penalizes the discrepancy between $\mathbf{f}$ and the observations $\left(\mathbf{y}^{(1)}, \mathbf{y}^{(2)}\right)$, according to the forward model (2). In the presence of white Gaussian noise, the latter term is usually taken, in its penalized form, as a squared $\ell_{2}$ distance, leading to an optimization problem of the form

$$
\underset{\mathbf{f} \in \mathbb{R}^{n}}{\operatorname{minimize}} R(\mathbf{f})+\lambda_{1}\left\|\mathbf{S H f}-\mathbf{y}^{(1)}\right\|^{2}+\lambda_{2}\left\|\mathbf{M f}-\mathbf{y}^{(2)}\right\|^{2} .
$$

When the noise level in the observed HR part can be neglected, or if one does not want to modify the observed HR part, one can instead consider the constrained problem

$$
\underset{\mathbf{f} \in \mathbb{R}^{n}}{\operatorname{minimize}} R(\mathbf{f})+\lambda\left\|\mathbf{S H f}-\mathbf{y}^{(1)}\right\|^{2}+\iota_{\left\{\mathbf{M} \cdot=\mathbf{y}^{(2)}\right\}}(\mathbf{f}),
$$

where we write $\{\mathbf{A} \cdot=\mathbf{y}\}$ for the set $\left\{\mathbf{x} \in \mathbb{R}^{n}: \mathbf{A x}=\mathbf{y}\right\}, \iota_{C}$ is the indicator function of a convex set $C$, given by $\iota_{C}(x)=0$ if $x \in C$ and $\iota_{C}(x)=+\infty$ otherwise, and $\lambda>0$. This latter setting is the one we adopt in the rest of the paper.

To summarize, we tackle the IZC problem by minimizing an energy function of the form given in (4). The problem now amounts to devising suitable regularizers $R$ as well as the practical optimization of the corresponding energies.

\section{IZC VIA PROPOSED NONLOCAL REgUlaRIZATION}

\section{A. The Nonlocal Framework}

Classical variational techniques employed in the field of image processing rely on the regularity of the underlying image in terms of local relationships between neighboring pixels. Nonlocal regularization techniques [22], [23] replace this local regularity assumption by a nonlocal one, exploiting patch redundancy across the whole image. The large success of these methods stems from their ability to preserve textures, details and fine structures better than their local counterparts. This arises from the fact that nonlocal regularization strategies are generally based on discrete difference operators whose orientations are driven by the observed image itself. Such directions are chosen for each pixel independently, based on a given notion of similarity. Further references about the nonlocal approach, targeted specifically towards the regularization of inverse problems in image processing, can be found e.g. in [24], [25], [26], [27], [28], [29], [30], [31].

In the following, we will consider the $\ell_{1,2}$ nonlocal total variation (NLTV), expressed as

$$
\operatorname{NLTV}(\mathbf{f})=\sum_{i=1}^{n} \sqrt{\sum_{j \in \mathcal{N}_{i}} w_{i, j}\left\|f_{i}-f_{j}\right\|^{2}},
$$

where $\mathcal{N}_{i}$ is a subset of positions located inside a search window centered at $i$. For each pixel $i$, we design the support $\mathcal{N}_{i}$ by selecting $k$ pixels that are most similar to $i$ according to the Euclidean distance between surrounding patches. Letting $p_{i}(\mathbf{f}) \in \mathbb{R}^{q}$ denote a $\sqrt{q} \times \sqrt{q}$ image patch extracted from $\mathbf{f}$ and centered at $i$, the obtained edges are then weighted according to

$$
w_{i, j}=e^{-\left\|p_{i}(\mathbf{f})-p_{j}(\mathbf{f})\right\|^{2} / 2 \sigma^{2}},
$$

where $\sigma>0$. We note that the nearest-neighbors search leads to a nonlocal neighborhood relation which is not symmetric: a pixel $j$ can be a neighbor of $i\left(j \in \mathcal{N}_{i}\right)$ without $i$ being a neighbor of $j\left(i \notin \mathcal{N}_{j}\right)$.

\section{B. Nonlocal regularization for IZC}

In order to adopt a nonlocal strategy for the IZC problem, we first need to define a weighted graph encoding neighborhood relations between HR pixels. As only part of the HR image is sensed, the information about all HR patches is incomplete, and thus it cannot be used to build the graph. In order to circumvent this difficulty, we take advantage of the available LR image $\mathbf{y}^{(1)} \in \mathbb{R}^{p}$. The first step in this strategy is to interpolate $\mathbf{y}^{(1)} \in \mathbb{R}^{p}$ to match the definition of $\mathbf{y}^{(2)} \in \mathbb{R}^{n}$. To this end, we use bicubic interpolation and get $\tilde{\mathbf{y}}^{(1)} \in \mathbb{R}^{n}$. Once we have $\tilde{\mathbf{y}}^{(1)}$, the similarities between patches can be estimated by the following two different approaches.

The first approach is based on the hypothesis that the interpolated patches generally exhibit similar spectral structure and maintain the same coherence. So, we connect each pixel to its $k$-nearest neighbors, inside a search window, according to the Euclidean distance between surrounding patches. For the regularization, we use the function introduced in Eq. (5). Letting $\|\cdot\|_{1,2}$ denote the following norm

$$
\left(\forall \mathbf{p} \in \mathbb{R}^{n \times m}\right) \quad\|\mathbf{p}\|_{1,2}=\sum_{i=1}^{n}\left\|\mathbf{p}_{i, .}\right\|_{2}=\sum_{i=1}^{n} \sqrt{\sum_{j=1}^{m} p_{i, j}^{2}},
$$

the NLTV penalty can be expressed as

$$
\operatorname{NLTV}(\mathbf{f})=\left\|\mathbf{D}_{1} \mathbf{f}\right\|_{1,2},
$$

where

$$
\left.\mathbf{D}_{1} \mathbf{f}=\left[\begin{array}{c}
\left.\left.\left[\sqrt{w_{1, j}}\left(f_{1}-f_{j}\right)\right]_{j \in \mathcal{N}_{1}}\right]\right\} \\
\vdots \\
{\left[\sqrt{w_{n, j}}\left(f_{n}-f_{j}\right)\right]_{j \in \mathcal{N}_{n}}}
\end{array}\right]\right\} \in \mathbb{R}^{k} .
$$


The second approach consists in adding to the previous set of connections a new one. To do so, we denote the set of pixels where the HR information is missing by $D$ and the set of sensed HR pixels by $D^{c}$ (see Figure 1). We connect each unobserved HR pixel $i \in D$ to its $k$-nearest observed HR neighbors in $D^{c}$, again using patch-based distances. The corresponding nonlocal gradient operator is expressed as

$$
\left(\mathbf{D}_{2} \mathbf{f}\right)_{i, .}= \begin{cases}{\left[\sqrt{\tilde{w}_{i, j}}\left(f_{i}-y_{j}^{(2)}\right)\right]_{j \in \tilde{\mathcal{N}}_{i}} \in \mathbb{R}^{k},} & \text { if } i \in D \\ \mathbf{0} \in \mathbb{R}^{k}, & \text { otherwise, }\end{cases}
$$

where $\mathbf{A}_{i, \text {. denotes the }} i^{\text {th }}$ line of a matrix $\mathbf{A}, \tilde{\mathcal{N}}_{i}$ is the set of nearest neighbors in $D^{c}$ of $i \in D$, and $\tilde{w}_{i, j}$ denotes the weight computed between patches $p_{i}\left(\tilde{y}^{(1)}\right)$ and $p_{j}\left(\tilde{y}^{(1)}\right)$. Therefore, the second regularizer we propose is defined in terms of $\mathbf{D}=\left[\begin{array}{ll}\mathbf{D}_{1}^{\top} & \mathbf{D}_{2}^{\top}\end{array}\right]^{\top}$, leading to

$$
\operatorname{NLTV}^{+}(\mathbf{f})=\|\mathbf{D f}\|_{1,2}
$$

Incorporating data fidelity terms as in Eq. (4) leads to the problem

$$
\underset{\mathbf{f} \in \mathbb{R}^{n}}{\operatorname{minimize}}\|\mathbf{D f}\|_{1,2}+\frac{\lambda}{2}\left\|\mathbf{S H} \mathbf{f}-\mathbf{y}^{(1)}\right\|^{2}+\iota_{\left\{\mathbf{M} \cdot=\mathbf{y}^{(2)}\right\}}(\mathbf{f}) .
$$

To summarize, we use an interpolated version of the LR image to construct a weighted adjacency relations and use it to infer the values of the missing pixels by solving (12).

\section{Minimization Using FBPD}

Problem (12) can be solved using proximal algorithms. In this paper, we use the forward-backward primal-dual (FBPD) algorithm of [19] with the following identifications: $F \equiv$ $\frac{\lambda}{2}\left\|\mathbf{S H} \cdot-\mathbf{y}^{(1)}\right\|^{2}, G \equiv \iota_{\left\{\mathbf{M} \cdot=\mathbf{y}^{(2)}\right\}}, H \equiv\|\cdot\|_{1,2}$ and $L=\mathbf{D}$. The authors might refer the appendix for relevant notations and definitions used.

In order to apply FBPD, we need to evaluate $\nabla F, \operatorname{prox}_{\tau G}$ and $\operatorname{prox}_{\omega H *}$ at each iteration. The gradient of $F$ is given by

$$
\nabla F(\mathbf{f})=\lambda \mathbf{H}^{\top} \mathbf{S}^{\top} \mathbf{S H f}-\lambda \mathbf{H}^{\top} \mathbf{S}^{\top} \mathbf{y}^{(1)},
$$

whose Lipschitz constant is equal to $\lambda$. $^{1}$

The evaluation of $\operatorname{prox}_{\tau G}$ amounts to projecting on the constraint set. It is given by

$$
\left(\operatorname{proj}_{\left\{\mathbf{M} \cdot=\mathbf{y}^{(2)}\right\}}(\mathbf{f})\right)_{i}= \begin{cases}y_{i}^{(2)} & \text { if } m_{i}=1, \\ f_{i} & \text { if } m_{i}=0 .\end{cases}
$$

The evaluation of $\operatorname{prox}_{\omega H^{*}}$ is given by

$$
\operatorname{prox}_{\omega H} *(y)=y-\operatorname{soft}_{1}(y),
$$

where soft is the vector-field soft-thresholding function given, for all $\mathbf{p} \in \mathbb{R}^{n \times m}$ and $\nu>0$ by

$$
\left(\operatorname{soft}_{\nu} \mathbf{p}\right)_{i, j}= \begin{cases}0 & \text { if }\left\|\mathbf{p}_{i, .}\right\|_{2} \leqslant \nu \\ \left(1-\frac{\nu}{\left\|\mathbf{p}_{i, .}\right\|}\right) p_{i, j} & \text { else. }\end{cases}
$$

\footnotetext{
${ }^{1}$ Since $\mathbf{S}^{\top} \mathbf{S}$ is diagonal with binary entries, we have that $\left\|\mathbf{S}^{\top} \mathbf{S}\right\|=1$. Using a normalized kernel $\mathbf{H}$ leads to $\left\|\mathbf{H}^{\top}\right\|=\|\mathbf{H}\|=1$, where $\|\cdot\|$ denotes the spectral norm.
}

The final point that needs investigation is the majoration of the operator norm of $\mathbf{D}$. Let us denote $w^{+}$the weights corresponding ${ }^{2}$ to $\mathbf{D}$. We have

$$
\begin{aligned}
\|\mathbf{D f}\|^{2} & =\sum_{i=1}^{n} \sum_{j \in \mathcal{N}_{i} \cup \tilde{\mathcal{N}}_{i}} w_{i, j}^{+}\left(f_{i}-f_{j}\right)^{2} \\
& \leqslant 2 \sum_{i=1}^{n} \sum_{j \in \mathcal{N}_{i} \cup \tilde{\mathcal{N}}_{i}} w_{i, j}^{+}\left(f_{i}^{2}+f_{j}^{2}\right),
\end{aligned}
$$

so that $\|D\| \leqslant 2 m$, where

$$
\begin{gathered}
m=\max _{i \in\{1 \ldots, n\}}\left(\operatorname{deg}_{i}^{\text {out }}+\operatorname{deg}_{i}^{\text {in }}\right), \\
\operatorname{deg}_{i}^{\text {out }}=\sum_{j \in \mathcal{N}_{i} \cup \tilde{\mathcal{N}}_{i}} w_{i, j}^{+} \text {and } \operatorname{deg}_{i}^{\text {in }}=\sum_{j \in \mathcal{N}_{i} \cup \tilde{\mathcal{N}}_{i}} w_{j, i}^{+} .
\end{gathered}
$$

Algorithm 1 summarizes all these computations. The variable of interest at convergence is $\mathrm{x}^{[l]}$.

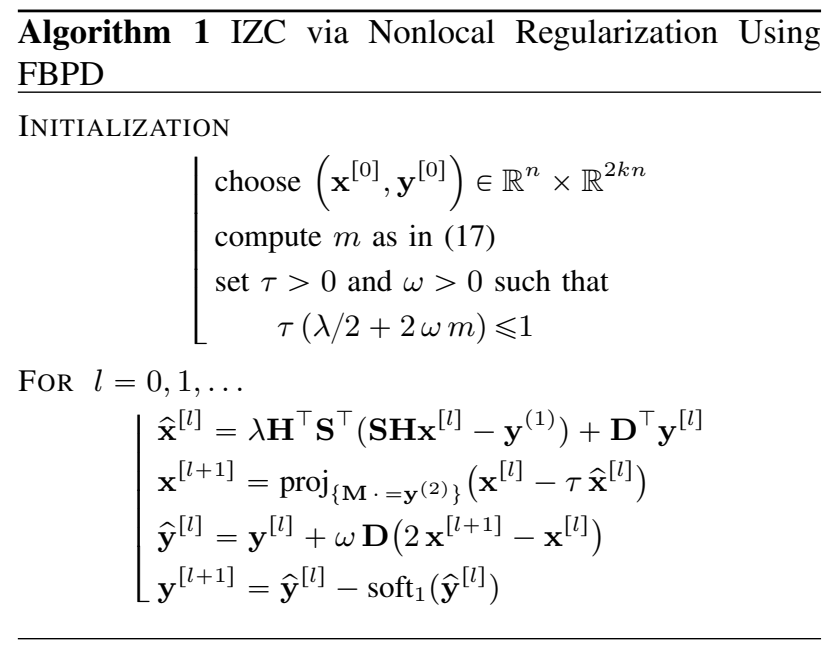

\section{Discussion}

The results obtained by the nonlocal approach we have just introduced are further discussed in Section V. In this subsection, we discuss the iteration complexity of Algorithm 1. We also discuss two foreseeable shortcomings of the proposed nonlocal approach.

From a computational point of view, the iteration complexity of the above algorithm is dominated by the application of two low-pass filters $\left(\mathbf{H}\right.$ and $\left.\mathbf{H}^{\top}\right)$ and the matrices $\mathbf{D}$ and $\mathbf{D}^{\top}$. The latter depends on the sparsity of $\mathbf{D}$, which is related to the number of unobserved HR pixels and to the number $k$ of nearest-neighbors. Furthermore, there is an overhead for performing nearest neighbors search.

As described earlier, patch similarities that drive the nonlocal regularization are based on (an interpolated version of) the LR image $y^{(1)}$. When the super-resolution factor $r$ is important and/or when the blurring matrix attenuates too much the high frequencies of the HR scene, these weights can be erroneous and their incorporation into the regularization functional can lead to bad reconstructions. While a solution

$$
{ }^{2} w_{i, j}^{+}=w_{i, j}+\tilde{w}_{i, j} \text { if } i \in D \text { and } j \in D^{c} \text {, and } w_{i, j}^{+}=w_{i, j} \text { otherwise. }
$$


to the first problem (large super-resolution factor) can consist in performing zoom completion recursively using a small SR factor, the only solution to the second problem (strong attenuation of high frequencies) is to recompute the weights after a fixed number of iterations.

Another case where the nonlocal approach we proposed may fail is when the typical patterns present in the visible HR part are different from the ones that make the invisible part. In this case, the nearest-neighbors graphs provides very limited information and any use of the corresponding weights may lead to bad reconstructions.

\section{IZC VIA STANDARD TV-BASED METHOdS}

The standard approach for edge-preserving image restoration consists in penalizing the total variation of the soughtafter image. We present in this section a Douglas-Rachfordbased optimization procedure for the $\mathrm{TV}-\ell_{2}$ regularization of the IZC problem where the HR data is involved through an equality constraint. We also present an algorithm for a TVbased decomposition model allowing to impose different priors on the structure and texture components of the sought-after image. The two algorithms presented in this section serve a baseline for the comparisons we perform in Section V. The details of the derivation are given in the appendix.

\section{A. IZC via TV Regularization}

Let

$$
\sigma:\{1, \ldots, \sqrt{n}\} \times\{1, \ldots, \sqrt{n}\} \rightarrow\{1, \ldots, n\}
$$

denote a pixel enumeration ${ }^{3}$. We consider the discrete gradient operator $\nabla: \mathbb{R}^{n} \rightarrow \mathbb{R}^{n \times 2}$ given, for $1 \leqslant k, l \leqslant \sqrt{n}-1$ by

$$
\begin{cases}(\nabla \mathbf{f})_{\sigma(l, k), 1} & =f_{\sigma(l, k+1)}-f_{\sigma(l, k)}, \\ (\nabla \mathbf{f})_{\sigma(l, k), 2} & =f_{\sigma(l+1, k)}-f_{\sigma(l, k)} .\end{cases}
$$

We adopt circular boundary conditions so that $-\nabla^{\top} \nabla$, corresponding to the 4-stencil discretization of the Laplacian operator, is diagonalized by the discrete Fourier transform.

We adopt the following definition for the discrete total variation of an image $\mathbf{f} \in \mathbb{R}^{n}$ :

$$
\mathrm{TV}(\mathbf{f})=\|\nabla \mathbf{f}\|_{1,2} .
$$

Adopting (20) as a prior for our IZC problem leads to the following convex optimization problem

$$
\underset{\mathbf{f} \in \mathbb{R}^{n}}{\operatorname{minimize}}\|\nabla \mathbf{f}\|_{1,2}+\frac{\lambda}{2}\left\|\mathbf{S H f}-\mathbf{y}^{(1)}\right\|^{2}+\iota_{\left\{\mathbf{M} \cdot=\mathbf{y}^{(2)}\right\}}(\mathbf{f}) .
$$

The energy in (21) is composite: it mixes the operators $\mathbf{M}, \mathbf{S}, \mathbf{H}$, and it contains two nonsmooth terms. While it is possible to apply the FBPD algorithm to solve Problem (21), we follow here the strategy suggested in [32] by using variable splitting and deploying the Douglas-Rachford algorithm in a product space.

Letting $\mathcal{H}=\mathbb{R}^{n \times 2} \times \mathbb{R}^{n} \times \mathbb{R}^{n}$ and $K=\{(\nabla \mathbf{f}, \mathbf{H f}, \mathbf{f}), \mathbf{f} \in$ $\left.\mathbb{R}^{n}\right\}$, we rewrite (21) in the form

$$
\underset{\mathbf{x}=(\mathbf{p}, \mathbf{u}, \mathbf{f}) \in \mathcal{H}}{\operatorname{minimize}} F_{1}(\mathbf{x})+F_{2}(\mathbf{x})
$$

\footnotetext{
${ }^{3}$ For simplicity of notations, we work with square images.
}

where

$$
F_{1}(\mathbf{x})=\|\mathbf{p}\|_{1,2}+\frac{\lambda}{2}\left\|\mathbf{S u}-\mathbf{y}^{(1)}\right\|^{2}+\iota_{\left\{\mathbf{M} \cdot=\mathbf{y}^{(2)}\right\}}(\mathbf{f}),
$$

and

$$
F_{2}(\mathbf{x})=\iota_{K}(\mathbf{p}, \mathbf{u}, \mathbf{f}) .
$$

In order to apply Douglas-Rachford, we need to evaluate $\operatorname{prox}_{F_{1}}$ and $\operatorname{prox}_{F_{2}}$ at each iteration. We note that the evaluation of the $\mathbf{p}$-component of $\operatorname{prox}_{F_{1}}$ is given the softthresholding operator of equation (16). Further details are given in the appendix. They lead to the iterations reported in Algorithm 2. The variable of interest at convergence is $f_{1}^{[l]}$.

We note that the $\mathbf{f}_{1}^{[l]}$ and $\mathbf{u}_{2}^{[l]}$ updates in Algorithm 2 can be done without inner loops since $\mathbf{A}_{1}$ is diagonalized by the Fourier transform and $\mathbf{A}_{\mathbf{2}}$ is diagonal.

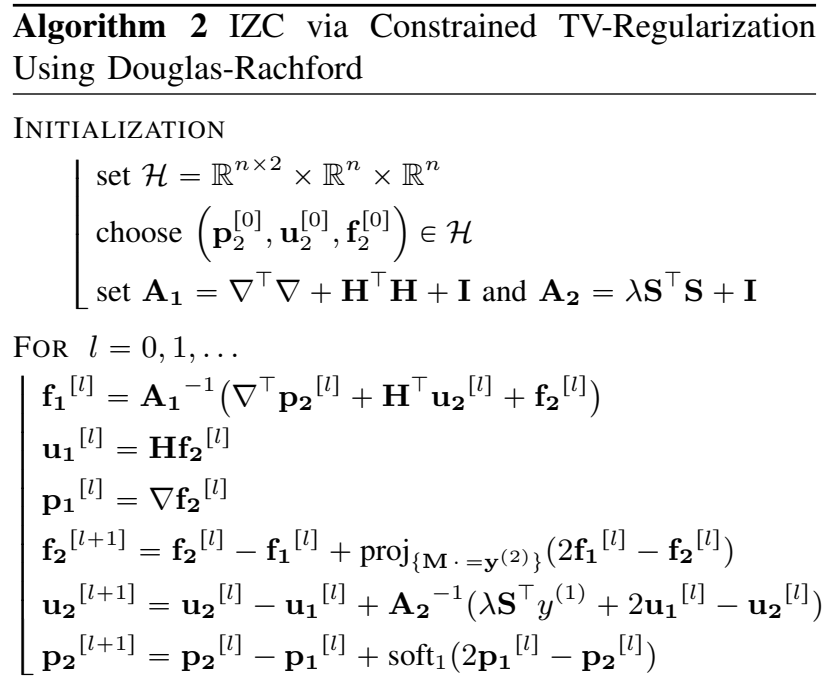

We show in Section $\mathrm{V}$ that the impact of this method, outside the HR visible area, is very limited. We next derive an algorithm based on a decomposition approach, allowing to partly circumvent this problem.

\section{B. IZC via $T V+$ Low-Patch-Rank Decomposition}

Rather than trying to impose a single prior for the soughtafter HR image, as was done in the previous section, we adopt here a decomposition model [33], [34] for the IZC problem. The idea is to compute separately two different components of the HR image by imposing different priors on each. The resulting image is obtained as the sum of these two components.

Of particular interest to us in the present section is the TV+low-patch-rank decomposition model of [20]. This model introduces a new formulation for the texture penalization term. More precisely, due to the repetitive aspect of natural textures, it is reasonable to assume that some patches of the texture part $\mathbf{v}$ of an image $\mathbf{f}$ will repeat themselves at different locations. In order to integrate this observation in a variational setting, the authors of [20] propose to extract all $\sqrt{q} \times \sqrt{q}$ nonoverlapping patches of an image $\mathbf{f} \in \mathbb{R}^{n}$, placing each of them as the column of a matrix $\mathcal{P} \mathbf{f} \in \mathbb{R}^{q \times m}$. Here, the patch-map 
$\mathcal{P}: \mathbb{R}^{n} \rightarrow \mathbb{R}^{q \times m}, n=q m$, allows to pass from an image f $\in \mathbb{R}^{n}$ to a matrix $\mathcal{P} \mathbf{f} \in \mathbb{R}^{q \times m}$ where $q$ is the number of pixels inside a patch and $m$ is the number of $\sqrt{q} \times \sqrt{q}$ nonoverlapping patches contained in $\mathbf{f}$. Let us note at this point that, due to the fact that $\mathcal{P}$ is essentially a permutation of image pixels, it is an isometry with respect to all point-wise norms on $\mathbb{R}^{q \times m}$. for deriving our minimization algorithm.

Equipped with the patch-map $\mathcal{P}$, the authors of [20] propose the following decomposition model

$$
\begin{aligned}
\underset{(\mathbf{u}, \mathbf{v}) \in \mathbb{R}^{n} \times \mathbb{R}^{n}}{\operatorname{minimize}} & \alpha \mathrm{TV}(\mathbf{u})+\beta\|\mathcal{P} \mathbf{v}\|_{*} \\
\text { subject to } & \mathbf{f}=\mathbf{u}+\mathbf{v},
\end{aligned}
$$

where $\|\mathbf{A}\|_{*}$ denotes the nuclear (or trace) norm of a matrix $\mathbf{A}$, that is, the sum of its singular values. In this context, the nuclear norm acts as a convex relaxation for the combinatorial rank function. Thus, adopting the term $\|\mathcal{P} \mathbf{v}\|_{*}$ favors an overall repetition of texture patches. Further details can be found in [20].

In [20], the decomposition model (25) is applied to denoising, deblurring and sparse reconstruction. The authors use the split Bregman algorithm [35] which is closely related to the algorithm we use in this paper. Let us finally note that a generalized version of the decomposition model of [20], where patches are allowed to overlap, has been recently proposed in [36].

Adapting (25) to our IZC setting leads to the following problem:

$$
\begin{aligned}
\underset{(\mathbf{u}, \mathbf{v}) \in \mathbb{R}^{n} \times \mathbb{R}^{n}}{\operatorname{minimize}} \quad \alpha \operatorname{TV}(\mathbf{u}) & +\beta\|\mathcal{P}(\mathbf{v})\|_{*}+\frac{\lambda}{2}\left\|\mathbf{S H}(\mathbf{u}+\mathbf{v})-\mathbf{y}^{(1)}\right\|^{2} \\
& +\iota_{\left\{\mathbf{M} \cdot=\mathbf{y}^{(2)}\right\}}(\mathbf{u}+\mathbf{v}),
\end{aligned}
$$

with $\alpha, \beta, \lambda>0$.

The advantage of using (26) instead of (21) is that the additional term $\|\mathcal{P}(\mathbf{v})\|_{*}$ allows to have better control on the texture part of the sought-after HR image. Let us also note that, due to the constraint imposed by the term $\iota_{\left\{\mathbf{M} \cdot=\mathbf{y}^{(2)}\right\}}(\mathbf{u}+\mathbf{v})$, this can be seen as an implicit strategy for making use of the available HR image in order to complete the unobserved part.

We carry out the minimization again by variable splitting and using the Douglas-Rachford algorithm. The splitting we adopt amounts to working in the Hilbert space $\mathcal{H}=\mathbb{R}^{n \times 2} \times$ $\mathbb{R}^{n} \times \mathbb{R}^{n} \times \mathbb{R}^{n} \times \mathbb{R}^{n}$. Letting

$$
K=\left\{(\nabla \mathbf{u}, \mathbf{u}, \mathbf{v}, \mathbf{H}(\mathbf{u}+\mathbf{v}), \mathbf{u}+\mathbf{v}) \in \mathcal{H}: \mathbf{u}, \mathbf{v} \in \mathbb{R}^{n}\right\},
$$

and for $\mathbf{x}=(\mathbf{p}, \mathbf{u}, \mathbf{v}, \mathbf{g}, \mathbf{w}) \in \mathcal{H}$

$$
\begin{aligned}
& F_{1}(\mathbf{x})=\alpha\|\mathbf{p}\|_{1,2}+\beta\|\mathcal{P}(\mathbf{v})\|_{*}+\frac{\lambda}{2}\left\|\mathbf{S g}-\mathbf{y}^{(1)}\right\|^{2} \\
& \quad+\iota_{\left\{\mathbf{M} \cdot=\mathbf{y}^{(2)}\right\}}(\mathbf{w}), \\
& F_{2}(\mathbf{x})=\iota_{K}(\mathbf{x}),
\end{aligned}
$$

Problem (26) is equivalent to minimizing $F_{1}+F_{2}$ over $\mathcal{H}$. In order to apply Douglas-Rachford, we need to evaluate prox $_{F_{1}}$ and $\operatorname{prox}_{F_{2}}$ at each iteration. The details are given in the appendix. We note that the evaluation of the $\mathbf{v}$-component of
$\operatorname{prox}_{F_{1}}$ involves the singular value thresholding operator given by:

$$
\operatorname{svt}_{\mu}(\mathbf{v})=\mathbf{U} \max (\boldsymbol{\Sigma}-\mu \mathbf{I}, 0) \mathbf{V}^{*},
$$

where $\mathbf{v}=\mathbf{U} \boldsymbol{\Sigma} \mathbf{V}^{*}$ is a singular value decomposition (SVD) of $\mathbf{v}$. We also note that the evaluation of $\operatorname{prox}_{F_{2}}$ leads to a system of linear equations whose matrix is

$$
\mathbf{A}_{3}=\left[\begin{array}{c|c}
\nabla^{\top} \nabla+\mathbf{H}^{\top} \mathbf{H}+2 \mathbf{I} & \mathbf{H}^{\top} \mathbf{H}+\mathbf{I} \\
\hline \mathbf{H}^{\top} \mathbf{H}+\mathbf{I} & \mathbf{H}^{\top} \mathbf{H}+2 \mathbf{I}
\end{array}\right] .
$$

Algorithm 3 summarizes the different steps. The variable of interest at convergence is $\mathbf{u}_{1}^{[l]}+\mathbf{v}_{1}^{[l]}$.

\begin{tabular}{l}
$\overline{\text { Algorithm } 3 \text { IZC via TV- }\|\mathcal{P} \cdot\|_{*} \text { Regularization Using }}$ \\
Douglas-Rachford \\
\hline
\end{tabular}

INITIALIZATION

$$
\left[\begin{array}{l}
\text { set } \mathcal{H}=\mathcal{H}=\mathbb{R}^{n \times 2} \times \mathbb{R}^{n} \times \mathbb{R}^{n} \times \mathbb{R}^{n} \times \mathbb{R}^{n} \\
\text { choose }\left(\mathbf{p}_{2}^{[0]}, \mathbf{u}_{2}^{[0]}, \mathbf{v}_{2}^{[0]}, \mathbf{g}_{2}^{[0]}, \mathbf{w}_{2}^{[0]}\right) \in \mathcal{H} \\
\text { set } \mathbf{A}_{3} \text { as in (30) and } \mathbf{A}_{2} \text { as in Algorithm 2 }
\end{array}\right.
$$

FOR $l=0,1, \ldots$

$$
\begin{aligned}
& {\left[\begin{array}{c}
\mathbf{u}_{1}^{[l]} \\
\mathbf{v}_{1}^{[l]}
\end{array}\right]=\mathbf{A}_{\mathbf{3}}{ }^{-1}\left[\begin{array}{c}
\nabla^{\top} \mathbf{p}_{\mathbf{2}}{ }^{[l]}+\mathbf{u}_{\mathbf{2}}{ }^{[l]}+\mathbf{H}^{\top} \mathbf{g}_{\mathbf{2}}{ }^{[l]}+\mathbf{w}_{\mathbf{2}}{ }^{[l]} \\
\mathbf{v}_{\mathbf{2}}{ }^{[l]}+\mathbf{H}^{\top} \mathbf{g}_{\mathbf{2}}{ }^{[l]}+\mathbf{w}_{\mathbf{2}}{ }^{[l]}
\end{array}\right]} \\
& \mathbf{g}_{\mathbf{1}}{ }^{[l]}=\mathbf{H}\left(\mathbf{u}_{\mathbf{1}}{ }^{[l]}+\mathbf{v}_{\mathbf{1}}{ }^{[l]}\right) \\
& \mathbf{w}_{\mathbf{1}}{ }^{[l]}=\mathbf{u}_{\mathbf{1}}{ }^{[l]}+\mathbf{v}_{\mathbf{1}}{ }^{[l]} \\
& \mathbf{p}_{\mathbf{1}}{ }^{[l]}=\nabla \mathbf{u}_{\mathbf{1}}{ }^{[l]} \\
& \mathbf{u}_{\mathbf{2}}{ }^{[l+1]}=\mathbf{u}_{\mathbf{1}}{ }^{[l]} \\
& \mathbf{v}_{\mathbf{2}}{ }^{[l+1]}=\mathbf{v}_{\mathbf{2}}{ }^{[l]}-\mathbf{v}_{\mathbf{1}}{ }^{[l]}+\mathcal{P}^{\top} \operatorname{svt}_{\beta} \mathcal{P}\left(2 \mathbf{v}_{\mathbf{1}}{ }^{[l]}-\mathbf{v}_{\mathbf{2}}{ }^{[l]}\right) \\
& \mathbf{g}_{\mathbf{2}}{ }^{[l+1]}=\mathbf{g}_{\mathbf{2}}{ }^{[l]}-\mathbf{g}_{\mathbf{1}}{ }^{[l]}+\mathbf{A}_{\mathbf{2}}{ }^{-1}\left(\lambda \mathbf{S}^{\top} y^{(1)}+2 \mathbf{g}_{\mathbf{1}}{ }^{[l]}-\mathbf{g}_{\mathbf{2}}{ }^{[l]}\right) \\
& \mathbf{w}_{\mathbf{2}}{ }^{[l+1]}=\mathbf{w}_{\mathbf{2}}{ }^{[l]}-\mathbf{w}_{\mathbf{1}}{ }^{[l]}+\operatorname{proj}_{\left\{\mathbf{M} \cdot=\mathbf{y}^{(2)}\right\}}\left(2 \mathbf{w}_{\mathbf{1}}{ }^{[l]}-\mathbf{w}_{\mathbf{2}}{ }^{[l]}\right) \\
& \mathbf{p}_{\mathbf{2}}{ }^{[l+1]}=\mathbf{p}_{\mathbf{2}}{ }^{[l]}-\mathbf{p}_{\mathbf{1}}{ }^{[l]}+\operatorname{soft}_{\alpha}\left(2 \mathbf{p}_{\mathbf{1}}{ }^{[l]}-\mathbf{p}_{\mathbf{2}}{ }^{[l]}\right)
\end{aligned}
$$

From a computational point of view, the iteration complexity of Algorithm 3 is dominated, on the one hand, by the computation of the singular value decomposition of the patch matrix, and on the other hand, by the solution of the linear system whose matrix is $\mathbf{A}_{3}$. Concerning the SVD computation, it is important to note that it applies to a matrix with much fewer rows (numbers of pixels in a patch) than columns (number of non-overlapping patches). Concerning the linear system, we note that while each block of $\mathbf{A}_{3}$ is diagonal in the Fourier domain, the overall matrix is not. We instead solve this linear system with a conjugate gradient method. In practice we perform only 4 iterations and use a 'warm start' strategy [37], meaning that we start the conjugate gradient solver at iteration $l+1$ with the result it yielded from iteration $l$. Each conjugate gradient iteration involves a matrix-vector product $\mathbf{A}_{3} \mathbf{x}$, so it involves the evaluation of one Laplacian filter $\left(\nabla^{\top} \nabla\right)$ and one low-pass filter $\left(\mathbf{H}^{\top} \mathbf{H}\right)$.

\section{EXPERIMENTAL RESUlts}

We start by evaluating the effectiveness of the methods we have introduced on a set of 4 natural images and 4 textures 
shown in Figure 2. These images correspond to $256 \times 256$ crops taken from the Kodak $^{4}$ and Brodatz ${ }^{5}$ databases.

In order to assess the relative performance of the different approaches, we start with a ground truth full HR image $\mathbf{f}_{0}$ and simulate the incomplete image $\mathbf{y}^{(2)}$ by masking $75 \%$ of $\mathbf{f}_{0}$. Similarly, we simulate the complete LR image $\mathbf{y}^{(1)}$ by blurring $\mathbf{f}_{0}$, downsampling the result by a factor $r$ in each direction, and finally adding white Gaussian noise. In all the experiments we carried, the blurring kernel corresponds to a normalized Gaussian $e^{-d^{2} / 2 s^{2}}$ with $s=1.2$. The kernel is truncated to a $5 \times 5$ window. The super-resolution factor $r$ is fixed to 2. The standard deviation of the additive white Gaussian noise is fixed to 2.5. Computations on images are done in the range $[0,255]$.

The results we report in Table I are obtained by empirically setting $\lambda=0.5$ in (12), $\lambda=2$ in (21), and $\alpha=0.5, \beta=$ 70 and $\lambda=5$ in (26). For the nonlocal approach detailed in Section III, three additional parameters are involved: the size of compared patches, the number $k$ of nearest neighbors, and the parameter $\sigma$ in (6). In our experiments, similarities between pixels were computed based on $5 \times 5$ surrounding patches inside a $25 \times 25$ search window. The number $k$ has been fixed to 14 , while $\sigma$ has been set in the interval $[10,50]$. Our nearest neighbors computation is performed exactly but we note that fast approximate computations can be performed, e.g. using the algorithm of [38]. For the TV+low-patch rank approach, a single additional parameter is involved: the size of the extracted patches. This value has been set to $64=$ $8 \times 8$ in all the experiments. A stopping criterion involving the relative change of two successive iterates has been applied for Algorithms 1, 2 and 3

Since, to the best of our knowledge, the IZC setting has not been considered in the literature (except in [2]), we cannot perform fair comparisons with other methods. Indeed, in the framework of SISR, there is no available HR data. However, we selected two state-of-the-art SISR methods whose implementations are freely available and decided to compare the performance yielded by our three algorithms with the ones yielded by these two. The methods we selected are those of Yang et al. [16] and of Dong et al. [14].

The method of [16] uses sparse code invariance of LR/HR patches over learned dictionaries. As the degradation model assumed in [16] is different from the one we adopted in this paper, we re-learned a dictionary of size 1024 form LR/HR examples synthesized from the same data set proposed in [16] but this time using our degradation model. We also adapted the back-projection step.

The method of Dong et al. [14] combines ideas from clustering and sparse coding with the nonlocal approach. In the first iteration of the algorithm, patches of an interpolated version of the LR image serve as a training set. They are clustered using K-means. A global dictionary is obtained, along with the corresponding sparse codes, by concatenating PCA sub-dictionaries from each cluster. The computed sparse codes are further refined by averaging with similar patches in

\footnotetext{
${ }^{4}$ http://r0k.us/graphics/kodak/

${ }^{5}$ Brodatz, P. (1966). Textures: A Photographic Album for Artists and Designers, Dover, New York.
}

a manner similar to the nonlocal-means approach [39], leading to sparse codes for each input interpolated patch. These sparse codes are further refined through a back-projection step aiming to enforce consistency with regard to the degradation model. A first estimation of the HR image is obtained by averaging overlapping patches. The overall procedure is iterated a fixed number of times, each time taking the output of the previous iteration as input for the next one.

In Table I we report PSNR and SSIM values between the 8 images of Figure 2 and the results obtained with the specific algorithms retained. These values are computed only on the reconstructed HR part. We also report the results obtained with bicubic interpolation. For each of our proposed methods, we also include reconstructions obtained using Algorithms 1,2 and 3 but without using the available HR data. This corresponds to using the same algorithms with the null matrix as a binary mask. The corresponding columns are labeled "no HR" in Table I. We also report the results obtained using the approach of [2], which corresponds to choosing $\mathbf{D}_{2}$ instead of D in (12).

As can be seen in Table I, the $\mathrm{NLTV}^{+}$achieves the best performance in terms of PSNR and SSIM. It is also interesting to note that the average PSNR and SSIM for $\mathrm{NLTV}^{+}$without using HR data is higher than that of [14]. Another interesting aspect is the average gain obtained by passing from the "no HR" to the "with HR" configuration. While this gain is very limited for the TV approach, it is more important for $\mathrm{TV}+\|\mathcal{P} \cdot\|_{*}$ and the nonlocal approach.

To further assess the performance of the proposed methods, we perform a second set of experiments using the 24 images of the Kodak database, in their original size $(768 \times 512$ or $512 \times$ $768)$. The parameters of the forward model remain unchanged. We report in Table II PSNR and SSIM values between the first ten images of the database and the reconstructions obtained using the retained method. The last row of Table II gives the average PSNR/SSIM values on the whole database. One can see here again that the proposed nonlocal approach yields the best performance. Finally, Table III summarizes the average running time of the different algorithms considered ${ }^{6}$.

The numerical evidence brought by Tables I and II can further be confirmed by inspecting the corresponding IZC reconstructions in Figures 3 and 4. Notice in particular how brick and wood edges are better reconstructed using $\mathrm{NLTV}^{+}$.

In Figure 5 we show the results obtained by our methods on a slice of a CT scan of a rock ${ }^{7}$. Therein, the zoom completion performed by $\mathrm{NLTV}^{+}$is hardly distinguishable from the true sensed HR image.

Finally, we show in Figures 6 and 7 the results we obtain on two $512 \times 512$ natural images. The setting is the same as for the previous experiments, except that the proportion of visible HR pixels is higher. On can see again that our proposed nonlocal method yields very satisfactory reconstructions.

\footnotetext{
${ }^{6}$ All algorithms were implemented in Matlab and executed on $2.9 \mathrm{GHz}$ CPU. For the methods of [16] and [14], we used the implementations available at the authors web pages.

${ }^{7}$ The authors would like to thank the team "Sismage" from the Group TOTAL for providing CT data.
} 


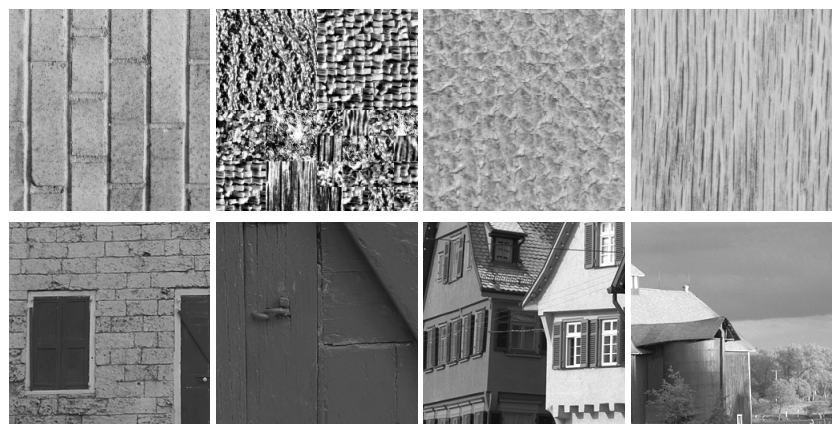

Fig. 2. Image dataset used as input to SR algorithms. From left to right and from top to bottom, $256 \times 256$ central crops of: "Brick", "Mosaic", "Pigskin", "Wood", "Kodim01", "Kodim02", "Kodim08", and "Kodim22".

TABLE I

PSNR (DB) AND SSIM ON THE RECONSTRUCTED HR AREA FOR DIFFERENT SR METHODS (IMAGES OF SIZE $256 \times 256$ )

\begin{tabular}{|c|c|c|c|c|c|c|c|c|c|c|c|c|c|}
\hline & Bicubic & & $r \mathbf{V}^{+}$ & & TV & Appro & $h$ of [2] & & $\mathrm{V}$ & $\mathrm{TV}+$ & $P \cdot \|_{*}$ & [16] & [14] \\
\hline & & no HR & with HR & no HR & with HR & no HR & with HR & no HR & with HR & no HR & with HR & & \\
\hline & 25.38 & 27.71 & 27.79 & 27.26 & 27.27 & 27.45 & 27.42 & 27.29 & 27.33 & 27.44 & 27.43 & 27.12 & 27.41 \\
\hline Brick & 0.616 & 0.697 & 0.847 & 0.639 & 0.640 & 0.698 & 0.846 & 0.692 & 0.763 & 0.711 & 0.777 & 0.711 & 0.719 \\
\hline Mosaic & 15.62 & 19.38 & 19.51 & 19.10 & 19.40 & 19.05 & 19.25 & 18.90 & 18.93 & 19.19 & 19.25 & 18.99 & 19.27 \\
\hline 10rosare & 0.589 & 0.811 & 0.909 & 0.805 & 0.910 & 0.799 & 0.904 & 0.818 & 0.862 & 0.835 & 0.876 & 0.827 & 0.845 \\
\hline Pioskin & 26.27 & 28.63 & 28.65 & 28.38 & 28.51 & 28.53 & 28.47 & 28.09 & 28.09 & 28.16 & 28.18 & 28.10 & 28.35 \\
\hline Pigskin & 0.660 & 0.799 & 0.902 & 0.788 & 0.901 & 0.801 & 0.903 & 0.775 & 0.824 & 0.794 & 0.838 & 0.799 & 0.805 \\
\hline Wood & 24.74 & 27.72 & 28.35 & 26.40 & 26.49 & 27.19 & 27.88 & 26.47 & 26.64 & 27.00 & 27.51 & 26.82 & 27.98 \\
\hline Wood & 0.624 & 0.801 & 0.907 & 0.794 & 0.794 & 0.779 & 0.899 & 0.746 & 0.795 & 0.747 & 0.804 & 0.759 & 0.804 \\
\hline Kodime1 & 24.09 & 26.29 & 26.71 & 26.22 & 26.23 & 26.00 & 26.17 & 26.19 & 26.21 & 26.32 & 26.35 & 26.09 & 26.62 \\
\hline Kodimur & 0.597 & 0.690 & 0.853 & 0.694 & 0.832 & 0.700 & 0.847 & 0.729 & 0.790 & 0.738 & 0.797 & 0.714 & 0.750 \\
\hline Kodim02 & 33.82 & 34.91 & 35.01 & 34.83 & 34.85 & 30.89 & 30.93 & 34.51 & 34.52 & 33.89 & 33.90 & 32.94 & 33.41 \\
\hline Kodim02 & 0.803 & 0.864 & 0.948 & 0.861 & 0.946 & 0.661 & 0.818 & 0.829 & 0.868 & 0.801 & 0.845 & 0.764 & 0.773 \\
\hline Kodim 08 & 21.59 & 25.64 & 25.97 & 25.41 & 25.42 & 24.84 & 24.96 & 25.29 & 25.32 & 25.37 & 25.40 & 24.17 & 25.91 \\
\hline Kodım08 & 0.657 & 0.804 & 0.904 & 0.802 & 0.897 & 0.744 & 0.865 & 0.796 & 0.839 & 0.796 & 0.839 & 0.765 & 0.812 \\
\hline Kodim 22 & 27.94 & 30.05 & 30.13 & 29.96 & 29.94 & 28.84 & 28.90 & 29.94 & 29.95 & 29.81 & 29.82 & 28.94 & 29.79 \\
\hline Kodim 22 & 0.771 & 0.846 & 0.918 & 0.845 & 0.916 & 0.713 & 0.849 & 0.823 & 0.867 & 0.800 & 0.839 & 0.766 & 0.780 \\
\hline & 24.93 & 27.54 & 27.76 & 27.19 & 27.26 & 26.59 & 26.74 & 27.08 & 27.12 & 27.14 & 27.23 & 26.64 & 27.34 \\
\hline Average & 0.664 & 0.789 & 0.898 & 0.778 & 0.854 & 0.736 & 0.866 & 0.776 & 0.826 & 0.777 & 0.826 & 0.763 & 0.786 \\
\hline
\end{tabular}

\section{CONCLUSION}

A super-resolution problem from an LR/HR image pair has been considered. We have motivated its importance and highlighted some of its possible applications. Then we developed a nonlocal regularization strategy for the construction of a solution and derived two TV-based algorithms. The practical optimization of the energies resulting from each model has been discussed.

While the TV-based solution is appropriate for the class of piecewise constant images, the nonlocal and the TV+lowpatch rank approaches allow targeting larger image classes, in particular images with strong repetitive textures. Both approaches organize the patches of the sought-after image following the evidence provided by the complete LR and incomplete HR image pair.

On average, the $\mathrm{TV}+\|\mathcal{P} \cdot\|_{*}$ method allows to go one step further than the standard TV- $\ell_{2}$ approach. However, we have seen that in order to truly leverage the available HR data, it is necessary to resort to explicit patch comparisons and to incorporate them in a nonlocal cost function.

We note that all the proposed methods do not rely on sparse representations over learned dictionaries and as such they can be easily adapted when the degradation model changes, e.g. when the blurring filter or the super-resolution factor changes.

Possible future work concerning the IZC problem, in particular when the blurring filter and the super-resolution factor are fixed, can concentrate on developing IZC strategies based on sparse and redundant representations [40]. As the setting for IZC is an LR/HR image pair, one can consider training both LR and visible HR patches in order to learn an adapted dictionary. When the super-resolution factor $r$ is important and/or the blurring matrix attenuates too much the high frequencies of the HR image, one can consider an iterative minimization process alternating between weight computation 


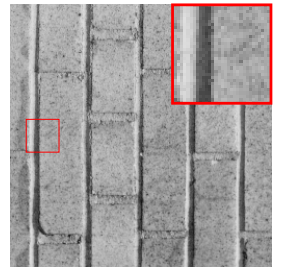

$f_{0}$

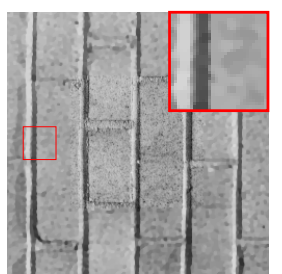

TV

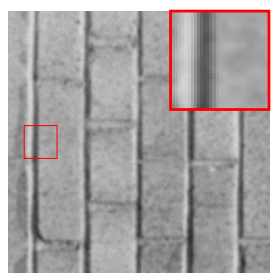

Bicubic

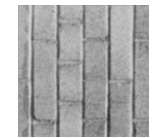

$y^{(1)}$

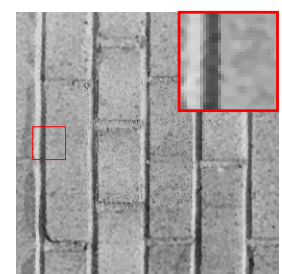

$\mathrm{TV}+\|\mathcal{P} \cdot\|_{*}$

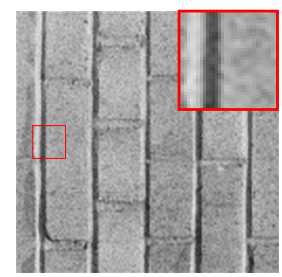

Yang et al.
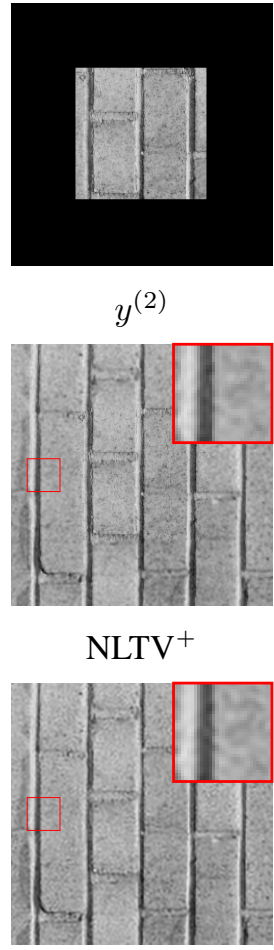

Dong et al.
Fig. 3. IZC for the image "Brick". From left to right and form top to bottom: ground-truth, LR input, incomplete HR input, TV $(\mathrm{psnr}=27.33), \mathrm{TV}+\|\mathcal{P} \cdot\|_{*}$ $(\mathrm{psnr}=27.43), \mathrm{NLTV}^{+}(\mathrm{psnr}=\mathbf{2 7 . 7 9})$, bicubic $(\mathrm{psnr}=25.38)$, Yang et al. [16] $(\mathrm{psnr}=27.12)$, Dong et al. [14] $(\mathrm{psnr}=27.41)$.

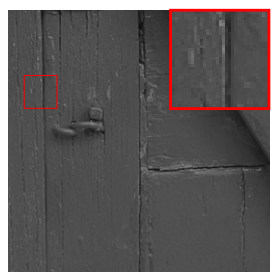

$f_{0}$

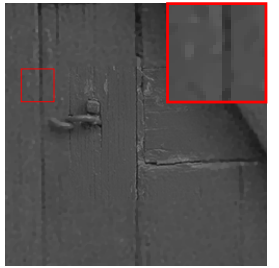

TV

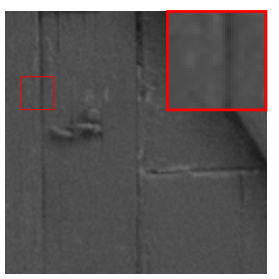

Bicubic

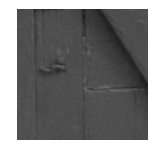

$y^{(1)}$

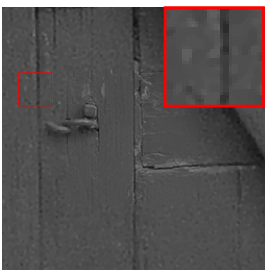

$\mathrm{TV}+\|\mathcal{P} \cdot\|_{*}$

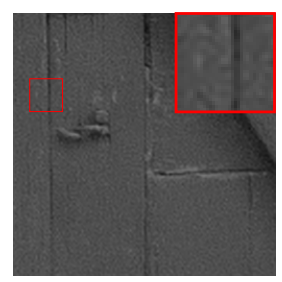

Yang et al.

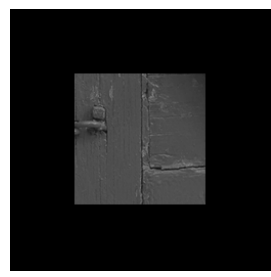

$y^{(2)}$

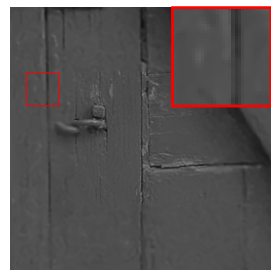

$\mathrm{NLTV}^{+}$

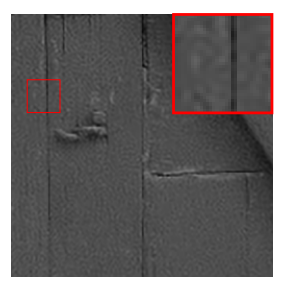

Dong et al.
Fig. 4. IZC for the image "Kodim02". From left to right and form top to bottom: ground-truth, LR input, incomplete HR input, TV $(\mathrm{psnr}=34.52)$, $\mathrm{TV}+\|\mathcal{P} \cdot\|_{*}(\mathrm{psnr}=33.90), \mathrm{NLTV}^{+}(\mathrm{psnr}=\mathbf{3 5 . 0 1})$, bicubic $(\mathrm{psnr}=33.82)$, Yang et al. [16] $(\mathrm{psnr}=32.94)$, Dong et al. [14] $(\mathrm{psnr}=33.41)$.
TABLE II

PSNR (DB) AND SSIM ON THE RECONSTRUCTED HR AREA FOR DIFFERENT SR METHODS (IMAGES OF SIZE $786 \times 512$ )

\begin{tabular}{|c|c|c|c|c|c|c|c|c|}
\hline & Bicubic & NLTV $^{+}$ & NLTV & [2] & TV & $\mathrm{TV}+\|\mathcal{P} \cdot\|_{*}$ & {$[16]$} & [14] \\
\hline & 23.47 & 26.21 & 25.94 & 25.55 & 25.34 & 26.04 & 25.47 & 26.27 \\
\hline Kodimu1 & 0.599 & 0.792 & 0.781 & 0.771 & 0.739 & 0.788 & 0.718 & 0.752 \\
\hline $\operatorname{dim} 02$ & 30.81 & .27 & 32.16 & 31.00 & 31.56 & 31.72 & 30.36 & 31.5 \\
\hline Ddimu2 & 0.774 & 0.852 & 0.849 & 0.785 & 0.832 & 0.825 & 0.686 & 0.764 \\
\hline $\operatorname{dim} 03$ & 31.29 & 33.70 & 33.43 & 31.49 & 33.01 & 32.87 & 30.90 & 32.69 \\
\hline (201mivs & 0.837 & 0.907 & 0.904 & 0.801 & 0.894 & 0.869 & 0.717 & 0.813 \\
\hline & 29.62 & 32.11 & 32.00 & 30.69 & 31.23 & 31.60 & 30.33 & 31. \\
\hline odim04 & 0.801 & 0.878 & 0.878 & 0.809 & 0.855 & 0.854 & 0.728 & 0.800 \\
\hline $\operatorname{dim} 05$ & 23.63 & & 27.00 & 26.49 & 26.10 & 26.93 & 26.50 & 27.12 \\
\hline Dermos & 0.702 & 0.870 & 0.865 & 0.825 & 0.828 & 0.857 & 0.794 & 0.823 \\
\hline & 25.51 & 27.97 & 27.76 & 26.92 & 27.21 & 27.82 & 26.92 & 27.90 \\
\hline & 0.664 & 0.826 & 0.823 & 0.731 & 0.792 & 0.813 & 0.698 & 0.753 \\
\hline & 30.09 & 34.72 & 34.25 & 31.07 & 33.77 & 33.32 & 31.22 & 32.92 \\
\hline odımo/ & 0.859 & 0.946 & 0.941 & 0.800 & 0.940 & 0.904 & 0.776 & 0.858 \\
\hline $\mathrm{m} 08$ & 20.88 & 24 & 24.03 & 23.79 & 23.28 & 23.87 & 23.49 & 24.26 \\
\hline Kodimus & 0.626 & 0.814 & 0.808 & 0.779 & 0.769 & 0.801 & 0.736 & 0.774 \\
\hline & 29.05 & 32.42 & 32.07 & 30.79 & 31.60 & 31.72 & 30.23 & 31.54 \\
\hline nodme & 0.824 & 0.907 & 0.905 & 0.815 & 0.901 & 0.873 & 0.722 & 0.813 \\
\hline & 28.28 & 31.4 & 31.10 & 29.44 & 30.24 & 30.64 & 29.58 & 31.08 \\
\hline Rodminto & 0.810 & 0.895 & 0.893 & 0.776 & 0.879 & 0.865 & 0.731 & 0.815 \\
\hline & 27.44 & & 29.95 & 28.67 & & & 28.55 & 29. \\
\hline & 0.751 & 0.864 & 0.861 & 0.777 & 0.841 & 0.842 & 0.725 & 0.79 \\
\hline
\end{tabular}

TABLE III

AVERAGE RUNNING TIME IN SECONDS OF THE CONSIDERED SR METHODS (IMAGES OF SIZE $768 \times 512$ )

$$
\begin{array}{cccccc}
\mathbf{N L T V}^{+} & \text {TV } & \text { TV+ }+\|\mathcal{P} \cdot\| * & {[16]} & {[14]} \\
402 & 30 & 221 & 562 & 1812
\end{array}
$$

and nonlocal regularization as was done in [41] for image inpainting.

\section{APPENDIX}

We recall in this appendix some definitions and algorithms used for the numerical optimization of the energy functions appearing in the paper.

\section{Proximity operators}

Let $(\mathcal{H},\langle.,\rangle$.$) be a finite-dimensional inner-product space.$ The associated norm is denoted by $\|\|=.\sqrt{\langle., .\rangle}$. A function $f: \mathcal{H} \rightarrow]-\infty,+\infty]$ is said to be proper if its domain, $\operatorname{dom}(f)=\{x \in \mathcal{H}: f(x)<+\infty\}$, is nonempty. The function $f$ is said to be convex if its epigraph, epi $(f)=$ $\{(x, a) \in \mathcal{H} \times \mathbb{R}: f(x) \leqslant a\}$, is convex; it is said to be lower semicontinuous if epi $(f)$ is closed. The set of all proper convex and lower semicontinuous functions from $\mathcal{H}$ to 

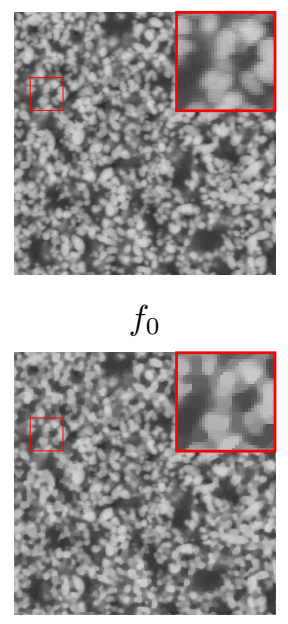

TV

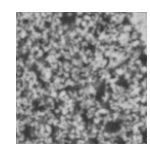

$y^{(1)}$

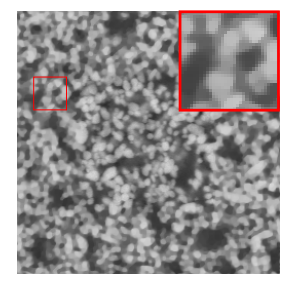

$\mathrm{TV}+\|\mathcal{P} \cdot\|_{*}$
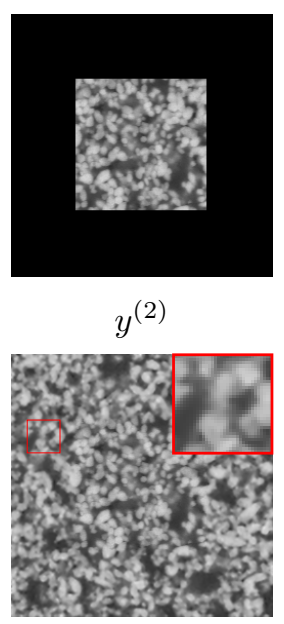

$\mathrm{NLTV}^{+}$
Fig. 5. IZC for a CT image scan. From left to right and from top to bottom : ground truth, LR image, partial HR image, TV-regularization $(\mathrm{psnr}=31.05)$, $\mathrm{TV}+\|\mathcal{P} \cdot\| *(\mathrm{psnr}=31.70), \mathrm{NLTV}^{+}(\mathrm{psnr}=\mathbf{3 2 . 1 7})$.

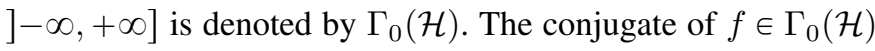
is the function $f^{*} \in \Gamma_{0}(\mathcal{H})$ defined for all $y \in \mathcal{H}$ by $f^{*}(y)=\sup _{x \in \mathcal{H}}\{\langle x, y\rangle-f(x)\}$.

For $f \stackrel{x \in \mathcal{H}}{\in} \Gamma_{0}(\mathcal{H})$ and $z \in \mathcal{H}$, the function $x \in \mathcal{H} \mapsto$ $\frac{1}{2}\|x-z\|^{2}+f(x)$ achieves its infimum at a unique point called proximity operator of $f$ at point $z$ and denoted by $\operatorname{prox}_{f} z$ :

$$
\operatorname{prox}_{f} z=\underset{x \in \mathcal{H}}{\operatorname{argmin}} f(x)+\frac{1}{2}\|x-z\|^{2} .
$$

For a nonempty closed $C \subset \mathcal{H}$ and $f=\iota_{C}$, we have that $f \in$ $\Gamma_{0}(\mathcal{H})$ and one recovers the definition of the Euclidean convex projection operator on $C$, denoted $\operatorname{proj}_{C}: \operatorname{proj}_{C} z=\operatorname{prox}_{\iota_{C}} z$.

An important property concerning proximal operators is their decomposability in orthonormal bases [21]. In particular, letting $\left(\mathcal{H}_{i}\right)_{1 \leqslant i \leqslant n}$ be a family of Hilbert spaces, $\mathcal{H}=\mathcal{H}_{1} \times$ $\ldots \times \mathcal{H}_{n}, f \in \Gamma_{0}(\mathcal{H}), f_{i} \in \Gamma_{0}\left(\mathcal{H}_{i}\right)$ for $i \in\{1, \ldots, n\}$, such that $f(x)=\sum_{i=1}^{n} f_{i}\left(x_{i}\right)$, we have $\left(\operatorname{prox}_{f}(x)\right)_{i}=\operatorname{prox}_{f_{i}}\left(x_{i}\right)$ for $i \in\{1, \ldots, n\}$.

\section{FBPD (Algorithm 3.1 of [19])}

Consider the following variational problem:

$$
\underset{x \in \mathcal{H}}{\operatorname{minimize}} F(x)+G(x)+H(L x)
$$

where

- $F$ is convex and differentiable with $\beta$-Lipschitz gradient;

- $L$ is linear from $\mathcal{H}$ to a finite dimensional inner-product space $\mathcal{X}$

- $G \in \Gamma_{0}(\mathcal{H})$ and $H \in \Gamma_{0}(\mathcal{X})$.

Suppose that the set of minimizers of Problem (32) is nonempty. Letting $\left(x_{n}\right)_{n}$ and $\left(y_{n}\right)_{n}$ constructed as follows

$$
\left\{\begin{array}{l}
x_{0} \in \mathcal{H}, y_{0} \in \mathcal{X}, \omega>0, \tau>0 \text { given } \\
x_{n+1}=\operatorname{prox}_{\tau G}\left(x_{n}-\tau\left(\nabla F\left(x_{n}+L^{\top} y_{n}\right)\right)\right) \\
y_{n+1}=\operatorname{prox}_{\omega H^{*}}\left(y_{n}+\omega L\left(2 x_{n+1}-x_{n}\right)\right) .
\end{array}\right.
$$

If $\tau\left(\lambda / 2+\omega\|L\|^{2}\right) \leqslant 1$, then sequence $\left(x_{n}\right)$ converges to a minimizer $x^{*}$ of Problem (32).

\section{Douglas-Rachford}

Consider the following variational problem:

$$
\underset{x \in \mathcal{H}}{\operatorname{minimize}} F_{1}(x)+F_{2}(x),
$$

with $F_{1}, F_{2} \in \Gamma_{0}(\mathcal{H})$. Let $\left(x_{n}\right)_{n},\left(y_{n}\right)_{n} \in \mathcal{H}^{\mathbb{N}}$ constructed as follows

$$
\left\{\begin{array}{l}
y_{0} \in \mathcal{H} \\
x_{n}=\operatorname{prox}_{\nu F_{2}}\left(y_{n}\right) \\
y_{n+1}=y_{n}+\xi\left(\operatorname{prox}_{\nu F 1}\left(2 x_{n}-y_{n}\right)-x_{n}\right)
\end{array}\right.
$$

with $0<\xi<2$ and $\nu>0$. If the set of minimizers of $F_{1}+F_{2}$ over $\mathcal{H}$ is nonempty, then the sequence $\left(x_{n}\right)$ converges to a minimizer $x^{*}$ of $F_{1}+F_{2}$ over $\mathcal{H}$. In all derivations, we set $\xi=\nu=1$.

\section{Derivation of Algorithm 2}

Letting $\mathcal{H}=\mathbb{R}^{n \times 2} \times \mathbb{R}^{n} \times \mathbb{R}^{n}$ and $K=\{(\nabla \mathbf{f}, \mathbf{H f}, \mathbf{f}), \mathbf{f} \in$ $\left.\mathbb{R}^{n}\right\}$, we rewrite (21) in the form (34) with $F_{1}$ given in (23) and $F_{2}$ given in (24). In order to apply Douglas-Rachford, we need to evaluate $\operatorname{prox}_{F_{1}}$ and $\operatorname{prox}_{F_{2}}$ at each iteration.

Since $F_{1}$ is separable, the evaluation of $\operatorname{prox}_{F_{1}}(\mathbf{p}, \mathbf{u}, \mathbf{f})$ amounts to evaluating each part separately [21]. The pcomponent corresponds to the vector-field soft-thresholding function given in (16). The evaluation of the $\mathbf{u}$-component amounts to solving a system of linear equations whose matrix is given by $\mathbf{A}_{2}=\lambda \mathbf{S}^{\top} \mathbf{S}+\mathbf{I}$. This computation can be done in linear time since $\mathbf{A}_{2}$ is diagonal. Finally, the evaluation of the $\mathbf{f}$-component is given in (14).

The evaluation of $\operatorname{prox}_{F_{2}}$ amounts to projecting on the constraint set $K$. It is straightforward to see that it leads to a system of linear equations whose matrix is $\mathbf{A}_{1}=\nabla^{\top} \nabla+$ $\mathbf{H}^{\top} \mathbf{H}+\mathbf{I}$. Due to the circular boundary choice for both $\nabla$ and $\mathbf{H}$, the matrix $\mathbf{A}_{1}$ is diagonalized by the discrete Fourier transform and thus the system can be solved by applying the discrete Fourier transform, modulating, and applying the inverse transform.

\section{Derivation of Algorithm 3}

Letting this time $\mathcal{H}=\mathbb{R}^{n \times 2} \times \mathbb{R}^{n} \times \mathbb{R}^{n} \times \mathbb{R}^{n} \times \mathbb{R}^{n}$ and $K=\left\{(\nabla \mathbf{u}, \mathbf{u}, \mathbf{v}, \mathbf{H}(\mathbf{u}+\mathbf{v}), \mathbf{u}+\mathbf{v}) \in \mathcal{H}: \mathbf{u}, \mathbf{v} \in \mathbb{R}^{n}\right\}$, we rewrite Problem (26) in the form (34) with $F_{1}$ and $F_{2}$ given in (27) and (28). In order to apply Douglas-Rachford, we need to evaluate $\operatorname{prox}_{F_{1}}$ and $\operatorname{prox}_{F_{2}}$ at each iteration.

As before, $F_{1}$ is separable, thus the evaluation of $\operatorname{prox}_{F_{1}}(\mathbf{p}, \mathbf{u}, \mathbf{v}, \mathbf{g}, \mathbf{w})$ amounts to evaluating each part separately. The evaluation of the p-component corresponds to the soft-thresholding function defined in (16). The u-component corresponds to the identity since $F_{1}$ does not depend on $\mathbf{u}$. The evaluation of the g-component involves solving a linear system with a diagonal matrix. The evaluation of the $\mathbf{w}$-component is given in (14).

The evaluation of the proximity operator of the $\mathbf{v}$ component is more intricate since it involves the precomposition with $\mathcal{P}$ and the nuclear norm. The generic problem is to compute $\operatorname{prox}_{\mu\|\mathcal{P} \cdot\|_{*}}$ for $\mu>0$. Due the fact that $\mathcal{P}$ 


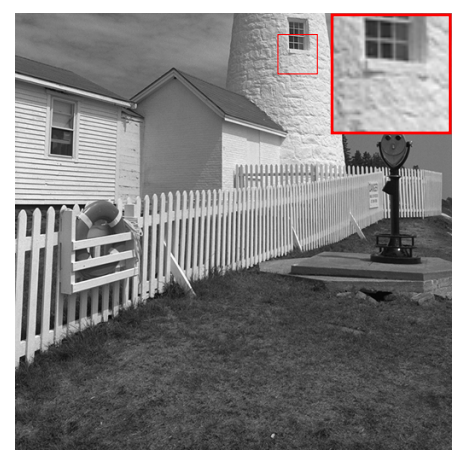

$f_{0}$

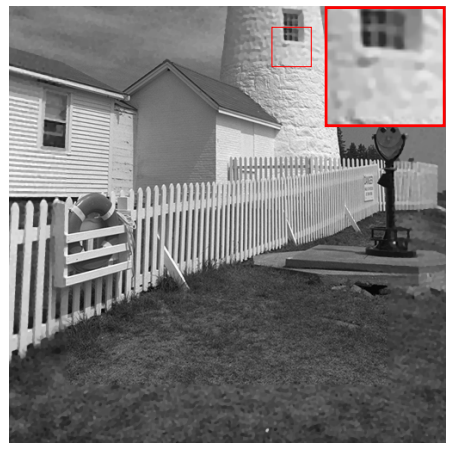

TV

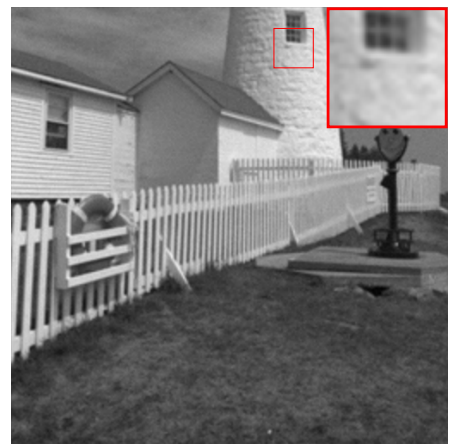

Bicubic

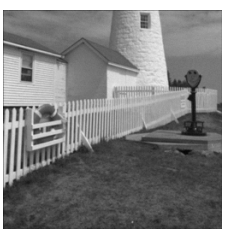

$y^{(1)}$

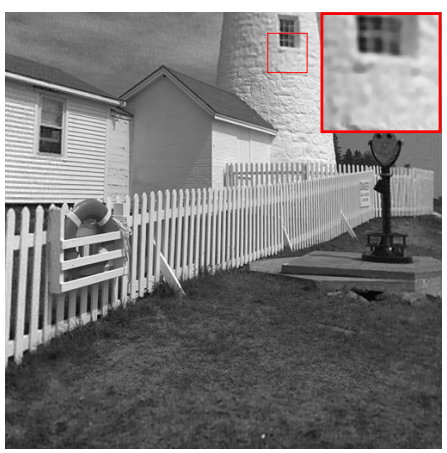

$\mathrm{TV}+\|\mathcal{P} \cdot\|_{*}$

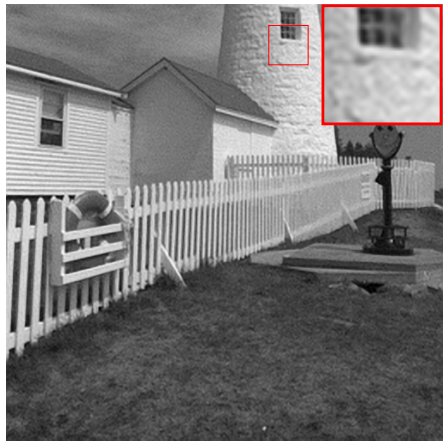

Yang et al.

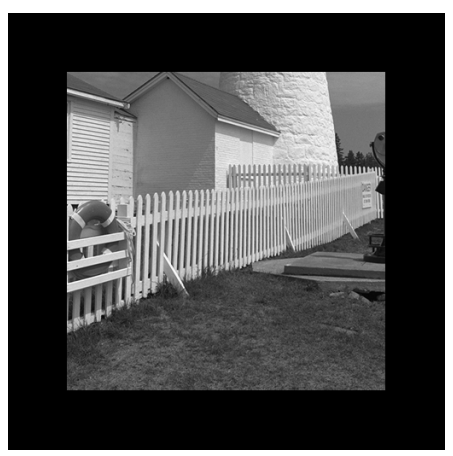

$y^{(2)}$

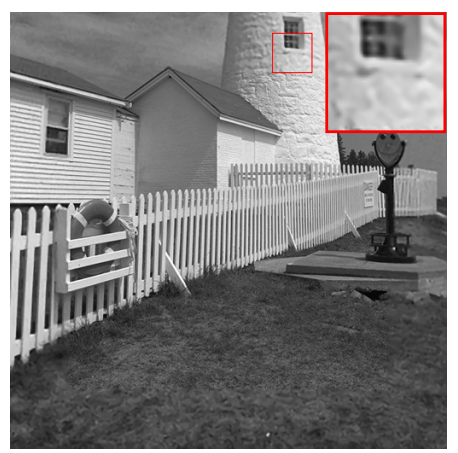

$\mathrm{NLTV}^{+}$

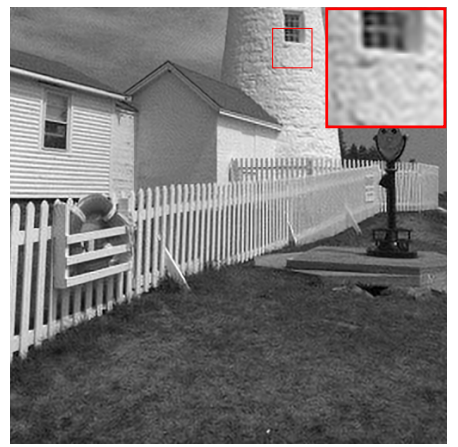

Dong et al.

Fig. 6. IZC for the image "Kodim19". From left to right and from top to bottom: ground truth, LR image, partial HR image, TV-regularization (psnr = 26.96), $\mathrm{TV}+\|\mathcal{P} \cdot\|_{*}(\mathrm{psnr}=27.01), \mathrm{NLTV}^{+}(\mathrm{psnr}=\mathbf{2 8 . 0 2})$, bicubic $($ psnr $=24.75)$, Yang et al. $($ psnr $=25.83)$, Dong et al. $(\mathrm{psnr}=27.26)$.

is an isometry with respect to all point-wise norms, we have (Proposition 11 of [21]) that

$$
\operatorname{prox}_{\mu\|\mathcal{P}(\cdot)\|_{*}}(\mathbf{v})=\mathcal{P}^{\top} \operatorname{prox}_{\mu\|\cdot\|_{*}} \mathcal{P}(\mathbf{v}) .
$$

It has been shown in [42] that the latter prox computation can be done by singular value thresholding:

$$
\operatorname{prox}_{\mu\|\cdot\|_{*}}(\mathbf{v})=\operatorname{svt}_{\mu}(\mathbf{v})=\mathbf{U} \max (\boldsymbol{\Sigma}-\mu \mathbf{I}, 0) \mathbf{V}^{*},
$$

where $\mathbf{v}=\mathbf{U} \boldsymbol{\Sigma} \mathbf{V}^{*}$ is a singular value decomposition (SVD) of $\mathbf{v}$.

Regarding the evaluation of $\operatorname{prox}_{F_{2}}$, it is easy to see that it leads to a system of linear equations with matrix $\mathbf{A}_{3}$ given in (30).

\section{REFERENCES}

[1] M. Bertero and P. Boccacci, Introduction to inverse problems in imaging. CRC press, 2010
[2] M. Hidane, J.-F. Aujol, Y. Berthoumieu, and C.-A. Deledalle, "Superresolution from a low- and partial high-resolution image pair," in International Conference on Image Processing, 2014, pp. 2145-2149.

[3] J. Yang and T. Huang, "Image super-resolution: Historical overview and future challenges," Super-resolution imaging, pp. 20-34, 2010.

[4] F. Malgouyres and F. Guichard, "Edge direction preserving image zooming: a mathematical and numerical analysis," SIAM Journal on Numerical Analysis, vol. 39, no. 1, pp. 1-37, 2001.

[5] L. Rudin, S. Osher, and E. Fatemi, "Nonlinear total variation based noise removal algorithms," Physica D, vol. 60, no. 1-4, pp. 259-268, 1992.

[6] M. Ebrahimi and E. R. Vrscay, "Solving the inverse problem of image zooming using self-examples?" in Image analysis and Recognition. Springer, 2007, pp. 117-130.

[7] G. Freedman and R. Fattal, "Image and video upscaling from local selfexamples," ACM Transactions on Graphics (TOG), vol. 30, no. 2, p. 12, 2011.

[8] D. Glasner, S. Bagon, and M. Irani, "Super-resolution from a single image," in Computer Vision, 2009 IEEE 12th International Conference on. IEEE, 2009, pp. 349-356.

[9] H. Takeda and P. Milanfar, "Locally adaptive kernel regression for spacetime super-resolution," Super-Resolution Imaging, Digital Imaging and Computer Vision, pp. 63-69, 2010. 


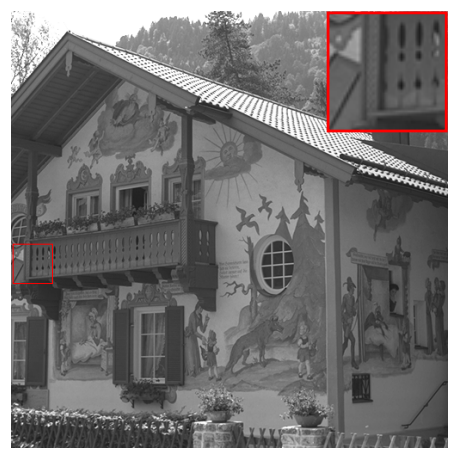

$f_{0}$

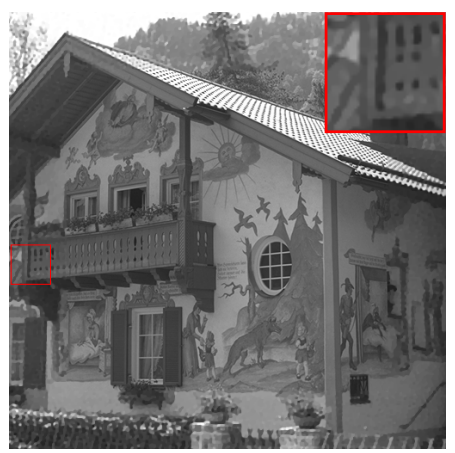

TV

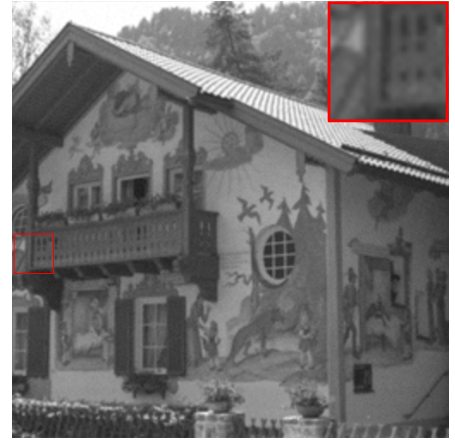

Bicubic

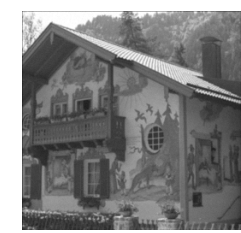

$y^{(1)}$

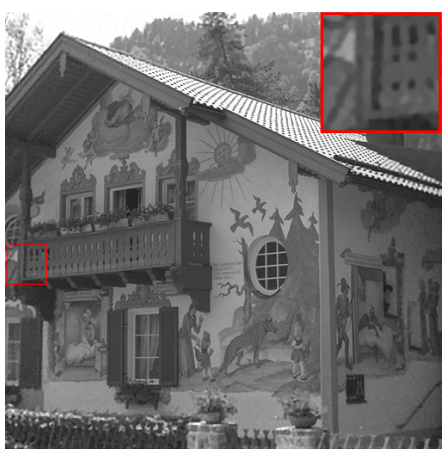

$\mathrm{TV}+\|\mathcal{P} \cdot\|_{*}$

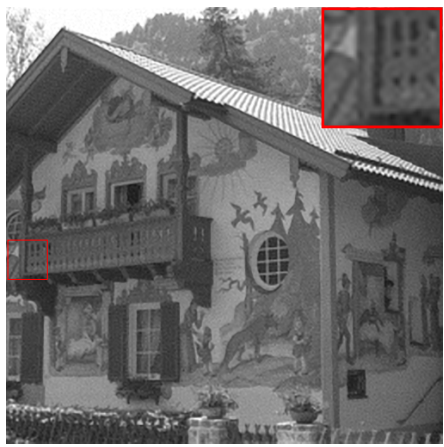

Yang et al.

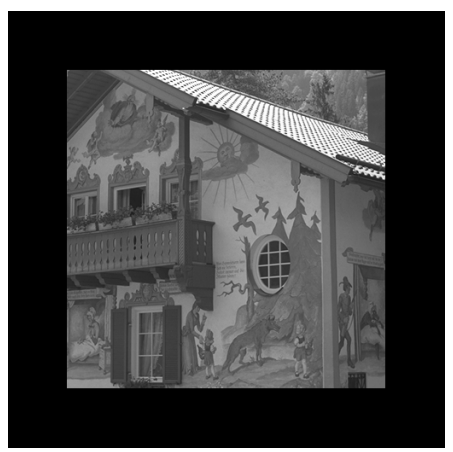

$y^{(2)}$

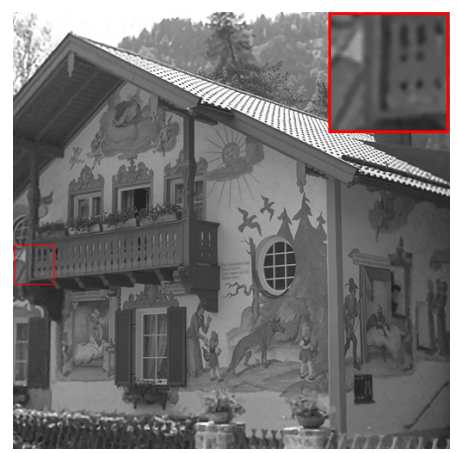

$\mathrm{NLTV}^{+}$

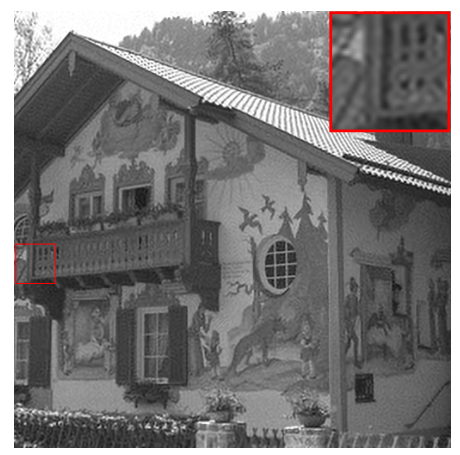

Dong et al.

Fig. 7. IZC for the image "Kodim24". From left to right and from top to bottom: ground truth, LR image, partial HR image, TV-regularization (psnr = $27.23), \mathrm{TV}+\|\mathcal{P} \cdot\|_{*}(\mathrm{psnr}=27.31), \mathrm{NLTV}^{+}(\mathrm{psnr}=\mathbf{2 7 . 5 7})$, bicubic $($ psnr $=24.78)$, Yang et al. $($ psnr $=26.64)$, Dong et al. $(\mathrm{psnr}=27.56)$.

[10] A. Adler, Y. Hel-Or, and M. Elad, "A shrinkage learning approach for single image super-resolution with overcomplete representations," in Computer Vision-ECCV 2010. Springer, 2010, pp. 622-635.

[11] S. Hawe, M. Kleinsteuber, and K. Diepold, "Analysis operator learning and its application to image reconstruction," Image Processing, IEEE Transactions on, vol. 22, no. 6, pp. 2138-2150, 2013.

[12] G. Yu, G. Sapiro, and S. Mallat, "Solving inverse problems with piecewise linear estimators: From gaussian mixture models to structured sparsity," Image Processing, IEEE Transactions on, vol. 21, no. 5, pp. 2481-2499, 2012.

[13] K. Zhang, X. Gao, D. Tao, and X. Li, "Multi-scale dictionary for single image super-resolution," in Computer Vision and Pattern Recognition (CVPR), 2012 IEEE Conference on. IEEE, 2012, pp. 1114-1121.

[14] W. Dong, L. Zhang, G. Shi, and X. Li, "Nonlocally centralized sparse representation for image restoration." IEEE Transactions on Image Processing, vol. 22, no. 4, pp. 1620-1630, 2013.

[15] K. Egiazarian and V. Katkovnik, "Single image super-resolution via bm3d sparse coding," in Signal Processing Conference (EUSIPCO), 2015 23rd European. IEEE, 2015, pp. 2849-2853.

[16] J. Yang, J. Wright, T. S. Huang, and Y. Ma, "Image super-resolution via sparse representation," Image Processing, IEEE Transactions on, vol. 19, no. 11 , pp. 2861-2873, 2010 .
[17] J. Yang, Z. Wang, Z. Lin, S. Cohen, and T. Huang, "Coupled dictionary training for image super-resolution," Image Processing, IEEE Transactions on, vol. 21, no. 8, pp. 3467-3478, 2012.

[18] R. Zeyde, M. Elad, and M. Protter, "On single image scale-up using sparse-representations," in Curves and Surfaces. Springer, 2010, pp. 711-730.

[19] L. Condat, "A primal-dual splitting method for convex optimization involving lipschitzian, proximable and linear composite terms," Journal of Optimization Theory and Applications, vol. 158, no. 2, pp. 460-479, 2013.

[20] H. Schaeffer and S. Osher, "A low patch-rank interpretation of texture," SIAM Journal on Imaging Sciences, vol. 6, no. 1, pp. 226-262, 2013.

[21] P. L. Combettes and J.-C. Pesquet, "A Douglas-Rachford splitting approach to nonsmooth convex variational signal recovery," Selected Topics in Signal Processing, IEEE Journal of, vol. 1, no. 4, pp. 564-574, 2007.

[22] G. Gilboa and S. Osher, "Nonlocal operators with applications to image processing," Multiscale Modeling \& Simulation, vol. 7, no. 3, pp. 10051028, 2008.

[23] A. Elmoataz, O. Lézoray, and S. Bougleux, "Nonlocal discrete regularization on weighted graphs: A framework for image and manifold processing," IEEE Transactions on Image Processing, vol. 17, no. 7, 
pp. 1047-1060, jul 2008.

[24] Y. Lou, X. Zhang, S. Osher, and A. Bertozzi, "Image recovery via nonlocal operators," Journal of Scientific Computing, vol. 42, no. 2, pp. 185-197, 2010.

[25] X. Zhang, M. Burger, X. Bresson, and S. Osher, "Bregmanized nonlocal regularization for deconvolution and sparse reconstruction," SIAM Journal on Imaging Sciences, vol. 3, no. 3, pp. 253-276, 2010.

[26] G. Peyré, S. Bougleux, L. D. Cohen et al., "Non-local regularization of inverse problems," Inverse Problems and Imaging, vol. 5, no. 2, pp. 511-530, 2011.

[27] A. Danielyan, V. Katkovnik, and K. Egiazarian, "Bm3d frames and variational image deblurring," Image Processing, IEEE Transactions on, vol. 21, no. 4, pp. 1715-1728, 2012.

[28] M. Hidane, O. Lézoray, and A. Elmoataz, "Nonlinear multilayered representation of graph-signals," Journal of mathematical imaging and vision, vol. 45, no. 2, pp. 114-137, 2013.

[29] C. Couprie, L. Grady, L. Najman, J.-C. Pesquet, and H. Talbot, "Dual constrained TV-based regularization on graphs," SIAM J. on Imaging Sciences, vol. 6, no. 3, pp. 1246-1273, Oct. 2013.

[30] G. Chierchia, N. Pustelnik, J.-C. Pesquet, and B. Pesquet-Popescu, "Epigraphical splitting for solving constrained convex optimization problems with proximal tools," J. on Image and Video Proc., 2014.

[31] J. Duran, A. Buades, B. Coll, and C. Sbert, "A nonlocal variational model for pansharpening image fusion," SIAM Journal on Imaging Sciences, vol. 7, no. 2, pp. 761-796, 2014.

[32] P. L. Combettes and J.-C. Pesquet, "A proximal decomposition method for solving convex variational inverse problems," Inverse problems, vol. 24, no. 6, p. 065014, 2008.

[33] J.-F. Aujol, G. Aubert, L. Blanc-Féraud, and A. Chambolle, "Image decomposition into a bounded variation component and an oscillating component," J. Math. Imaging Vis., vol. 22, no. 1, pp. 71-88, Jan. 2005.

[34] J.-F. Aujol and A. Chambolle, "Dual norms and image decomposition models," Int. J. Comput. Vision, vol. 63, no. 1, pp. 85-104, Jun. 2005.

[35] T. Goldstein and S. Osher, "The split bregman method for 11-regularized problems," SIAM Journal on Imaging Sciences, vol. 2, no. 2, pp. 323343, 2009.

[36] S. Ono, T. Miyata, and I. Yamada, "Cartoon-texture image decomposition using blockwise low-rank texture characterization," Image Processing, IEEE Transactions on, vol. 23, no. 3, pp. 1128-1142, March 2014.

[37] M. V. Afonso, J. M. Bioucas-Dias, and M. A. Figueiredo, "Fast image recovery using variable splitting and constrained optimization," Image Processing, IEEE Transactions on, vol. 19, no. 9, pp. 2345-2356, 2010.

[38] C. Barnes, E. Shechtman, D. B. Goldman, and A. Finkelstein, "The generalized PatchMatch correspondence algorithm," in European Conference on Computer Vision, Sep. 2010.

[39] A. Buades, B. Coll, and J.-M. Morel, "Image denoising methods. A new non-local principle," SIAM Review, vol. 52, no. 1, pp. 113-147, 2010.

[40] M. Elad, Sparse and redundant representations: from theory to applications in signal and image processing. Springer, 2010.

[41] P. Arias, G. Facciolo, V. Caselles, and G. Sapiro, "A variational framework for exemplar-based image inpainting," International journal of computer vision, vol. 93, no. 3, pp. 319-347, 2011.

[42] J.-F. Cai, E. J. Candès, and Z. Shen, "A singular value thresholding algorithm for matrix completion," SIAM Journal on Optimization, vol. 20, no. 4, pp. 1956-1982, 2010.

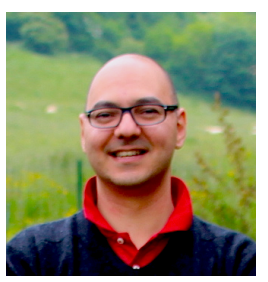

Moncef Hidane received the master's degree in applied mathematics from Université Paris 7 - Paris Diderot, France, in 2008, and the Ph.D. degree in computer science from Université de Caen Normandie, in 2013. He was a Post-Doctoral Fellow with Université de Bordeaux and is currently Associate Professor with the National Institute of Applied Sciences (INSA) Centre Val de Loire and member of the Laboratoire d'Informatique de l'Université François Rabelais, Tours, France. His research interests focus on variational approaches for image restoration and analysis.

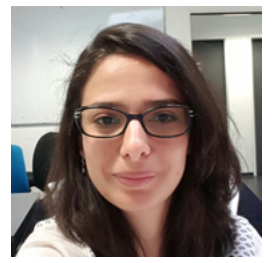

Mireille El Gheche received the engineering degree in Telecommunications from the Lebanese University (Tripoli, Lebanon) and the Master degree in Radio-communication from SupElec (Paris, France) in 2010. She received the Ph.D. degree in signal and image processing from the Université Paris Est (Marne-la-Vallée, France) in 2014. From 2015, she is a Postdoctoral Researcher at the IMS and IMB laboratories, Université de Bordeaux (Talence, France), where she is working on the topic of superresolution of texture images and texture volume for Computed Tomography applications. Her research interests are in convex and non-convex optimization applied to inverse problems, with a focus on image restoration, stereo matching, 3D reconstruction, and super-resolution.

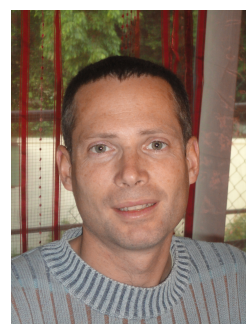

Jean-François Aujol studied Mathematics at the Ecole Normale Supérieure (ENS) in Cachan. He received the Ph.D. degree in Mathematics from Nice-Sophia-Antipolis University in June 2004 and the Habilitation thesis in Applied Mathematics from ENS Cachan in May 2009. In 2004-2005, he was successively an assistant researcher in UCLA (Applied Mathematics Department), and then a research engineer at Telecom ParisTech. In 2005-2011, JeanFrançois Aujol was a scientist researcher with CNRS at ENS Cachan with CMLA (until 2009), and then in Marseille with LATP. Since March 2011, he has been a full professor of applied mathematics in Bordeaux with IMB. Since September 2013, he has been a member of IUF (Institut Universitaire de France). He is interested in mathematical problems related to image processing. Jean-François Aujol serves as an associate editor for IEEE Transactions on Image Processing, Computer Vision and Image Understanding, and Journal of Mathematical Imaging and Vision. He is an IEEE member.

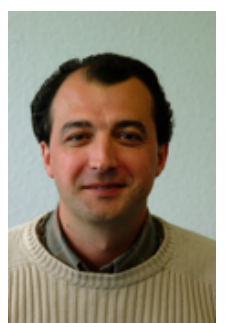

Yannick Berthoumieu received the $\mathrm{PhD}$ degree in January 1996 in signal processing from the University of Bordeaux I, France. In 1998, he joined the Ecole Nationale Supérieure dElectronique, Informatique et Radiocommunications de Bordeaux as an Associate Professor. He is currently a Full Professor with the Department of Telecommunications, Bordeaux National Institute of Technology. His major research interests are concerned with multidimensional signal analysis, stochastic modeling, texture analysis, and video processing. Since 2003 , he is with the Signal and Image processing group of IMS Lab, University of Bordeaux.

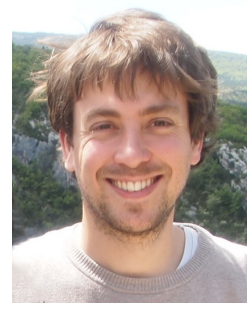

Charles-Alban Deledalle received the Engineer degree from EPITA, France, in 2008, the Master of Science and Technology degree from Université Paris VI, France, in 2008 and the Ph.D. degree in signal and image processing from Telecom ParisTech, France, in 2011. He made a postdoctoral fellowship in applied mathematics at Université Paris IX, France, in 2012. He is currently CNRS Researcher at Institut de Mathematiques de Bordeaux (IMB), Université de Bordeaux, France. His research interests include image denoising, reconstruction, variational and statistical modeling, and parameter estimation. He received the IEEE ICIP Best Student Paper Award in 2010, the ISIS/EEA/GRETSI Best PhD Award in 2012, and the IEEE GRSS Transactions Prize Paper Award in 2016. 